Prepared in cooperation with Yakima County, Washington, for the Lower Yakima River Basin Groundwater Management Area

\title{
Concentrations of Nitrate in Drinking Water in the Lower Yakima River Basin, Groundwater Management Area, Yakima County, Washington, 2017
}

Data Series 1084 
Cover: Yakama area drain site, Yakima River at Umantum, Washington (12484500), December 18, 2012. Photograph by Gabe Landrum, U.S. Geological Survey. 


\section{Concentrations of Nitrate in Drinking Water in the Lower Yakima River Basin, Ground water Management Area, Yakima County, Washington, 2017}

By Raegan L. Huffman

Prepared in cooperation with Yakima County, Washington, for the Lower Yakima

River Basin Groundwater Management Area

Data Series 1084 


\title{
U.S. Department of the Interior \\ RYAN K. ZINKE, Secretary
}

\author{
U.S. Geological Survey \\ James F. Reilly II, Director
}

U.S. Geological Survey, Reston, Virginia: 2018

For more information on the USGS - the Federal source for science about the Earth, its natural and living resources, natural hazards, and the environment-visit https://www.usgs.gov/ or call 1-888-ASK-USGS.

For an overview of USGS information products, including maps, imagery, and publications, visit https://store.usgs.gov.

Any use of trade, firm, or product names is for descriptive purposes only and does not imply endorsement by the U.S. Government.

Although this information product, for the most part, is in the public domain, it also may contain copyrighted materials as noted in the text. Permission to reproduce copyrighted items must be secured from the copyright owner.

Suggested citation:

Huffman, R.L., 2018, Concentrations of nitrate in drinking water in the lower Yakima River Basin, Groundwater Management Area, Yakima County, Washington, 2017: U.S. Geological Survey Data Series 1084, 18 p., https://doi. org/10.3133/ds1084.

ISSN 2327-638X (online) 


\section{Contents}

Abstract
Introduction
Description of Study Area
Sample Collection Design and Methods
Sites Selection
Sample Collection
Summary
References Cited

\section{Figures}

1. Map showing the location of the Groundwater Management Area and surficial hydrogeologic units, lower Yakima River Basin, Yakima County, Washington.

2. Map showing wells and drains sampled for nitrate in the lower Yakima River Basin, Groundwater Management Area, Yakima County, Washington, April-December 2017.

\section{Tables}

1. Location and description of groundwater wells sampled for nitrate in the Groundwater Management Area, Yakima County, Washington, April-December 2017.

2. Location of drains sampled for nitrate in the lower Yakima River Basin, Groundwater Management Area, Yakima County, Washington, April-December 2017.

3. Numbers of environmental and quality-assurance samples collected from wells and drains in the lower Yakima River Basin, Groundwater Management Area, Yakima County, Washington.

4. Nitrate concentrations in groundwater samples collected from drinking water wells in the lower Yakima River Basin, Groundwater Management Area, Yakima County, Washington, April-December, 2017

5. Nitrate concentrations in surface-water samples collected from drain sites in the lower Yakima River Basin, Groundwater Management Area, Yakima County, Washington, April-December 2017

6. Statistical summary of nitrate concentrations in groundwater and surface-water samples collected from domestic wells and drains in the lower Yakima River Basin, Groundwater Management Area, Yakima County, Washington. 


\section{Conversion Factors}

U.S. Customary Units to International System of Units

\begin{tabular}{|c|c|c|}
\hline Multiply & By & To obtain \\
\hline \multicolumn{3}{|c|}{ Length } \\
\hline foot $(\mathrm{ft})$ & 0.3048 & meter $(\mathrm{m})$ \\
\hline mile (mi) & 1.609 & kilometer $(\mathrm{km})$ \\
\hline \multicolumn{3}{|c|}{ Area } \\
\hline acre & 4,047 & square meter $\left(\mathrm{m}^{2}\right)$ \\
\hline acre & 0.4047 & hectare (ha) \\
\hline acre & 0.004047 & square kilometer $\left(\mathrm{km}^{2}\right)$ \\
\hline section ( 640 acres or 1 square mile) & 259.0 & square hectometer $\left(\mathrm{hm}^{2}\right)$ \\
\hline square mile $\left(\mathrm{mi}^{2}\right)$ & 259.0 & hectare (ha) \\
\hline square mile $\left(\mathrm{mi}^{2}\right)$ & 2.590 & square kilometer $\left(\mathrm{km}^{2}\right)$ \\
\hline \multicolumn{3}{|c|}{ Volume } \\
\hline acre-foot (acre-ft) & 1,233 & cubic meter $\left(\mathrm{m}^{3}\right)$ \\
\hline acre-foot (acre-ft) & 0.001233 & cubic hectometer $\left(\mathrm{hm}^{3}\right)$ \\
\hline \multicolumn{3}{|c|}{ Flow rate } \\
\hline acre-foot per year (acre-ft/yr) & 0.001233 & cubic hectometer per year $\left(\mathrm{hm}^{3} / \mathrm{yr}\right)$ \\
\hline
\end{tabular}

\section{Datums}

Vertical coordinate information is referenced to the North American Vertical Datum of 1988 (NAVD 88).

Horizontal coordinate information is referenced to the North American Datum of 1983 (NAD 83).

Altitude, as used in this report, refers to distance above the vertical datum.

\section{Supplemental Information}

Concentrations of chemical constituents in water are given in either milligrams per liter (mg/L) or micrograms per liter $(\mu \mathrm{g} / \mathrm{L})$.

Activities for radioactive constituents in water are given in picocuries per liter ( $\mathrm{pCi} / \mathrm{L})$ 


\section{Well-Numbering System}

In the State of Washington, wells and springs are assigned numbers that identify their location within a township, range, section, and 40 -acre tract. For example, local well number $10 \mathrm{~N} / 22 \mathrm{E}-15 \mathrm{~B} 01$ indicates that the well is in township 10 north and range 22 east, north and east of the Willamette Base Line and Meridian, respectively. The numbers immediately following the hyphen indicate the section (15) within the township; the letter (B) following the section gives the 40 -acre tract of the section. The two-digit sequence number (01) following the letter indicates that the well was the first one inventoried by U.S. Geological Survey personnel in that 40-acre tract. An "S," " $D$," or " $P$ " following the sequence number indicates that the site is a spring, deepened well, or piezometer in a well, respectively. In the figures of this report, wells and springs are identified individually by only the section and 40-acre tract, such as 15B01; township and range are shown on the map borders.

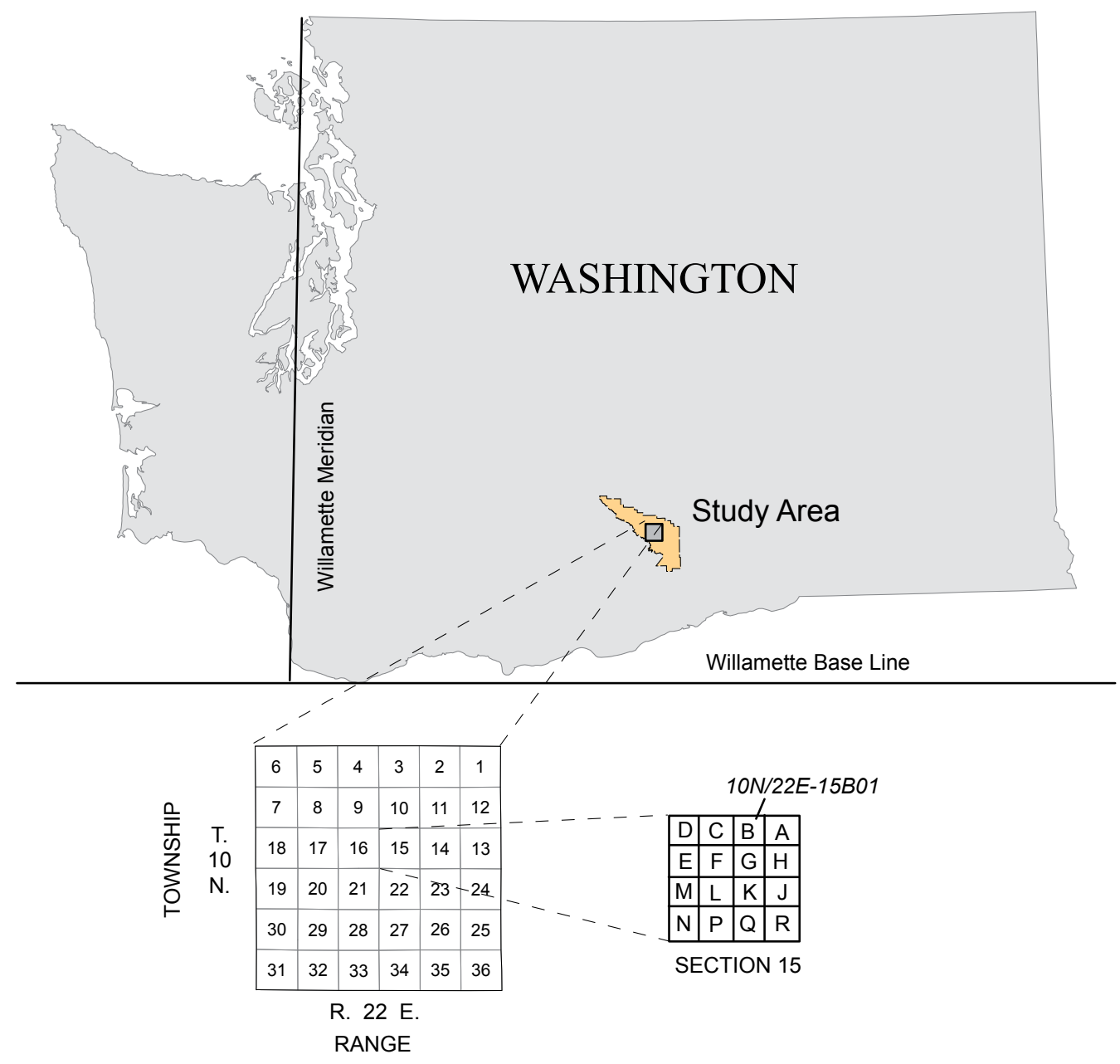





\title{
Concentrations of Nitrate in Drinking Water in the Lower Yakima River Basin, Groundwater Management Area, Yakima County, Washington, 2017
}

\author{
By Raegan L. Huffman
}

\begin{abstract}
The U.S. Geological Survey, in cooperation with the lower Yakima River Basin Groundwater Management Area (GWMA) group, conducted an intensive groundwater sampling collection effort of collecting nitrate concentration data in drinking water to provide a baseline for future nitrate assessments within the GWMA. About every 6 weeks from April through December 2017, a total of 1,059 samples were collected from 156 wells and 24 surface-water drains. The domestic wells were selected based on known location, completion depth, ability to collect a sample prior to treatment or filtration, and distribution across the GWMA. The drains were pre-selected by the GWMA group, and further assessed based on ability to access sites and obtain a representative sample.

More than 20 percent of samples from the domestic wells and 12.8 percent of drain samples had nitrate concentrations that exceeded the maximum contaminant level (MCL) of 10 milligrams per liter established by the U.S. Environmental Protection Agency. At least one nitrate concentration above the MCL was detected in 26 percent of wells and 33 percent of drains sampled. Nitrate was not detected in 13 percent of all samples collected.
\end{abstract}

\section{Introduction}

In the lower Yakima River Basin in central Washington State, 12-21 percent of private drinking-water wells have nitrate levels above the U.S. Environmental Protection Agency (EPA) Drinking Water Standard of 10 milligrams per liter (mg/L) (summarized in U.S. Environmental Protection Agency, 2012; and Washington State Department of Ecology 2010). The contaminated wells tend to be shallow and are primarily used to supply private residences.

Elevated levels of nitrate in drinking water in the lower Yakima River Basin are a high priority concern for a variety of governmental agencies and private groups, many of which are involved in the lower Yakima River Basin Groundwater Management Area (GWMA; fig. 1). The GWMA was formed in 2012 to identify ways to reduce nitrate concentrations in groundwater. In support of this goal, the GWMA has pursued a variety of approaches including public education campaigns to inform people about the problem of elevated nitrate levels, soil sampling to help farmers apply nutrients at agronomic rates, and the collection of groundwater nitrate samples to document the extent of the problem and provide a baseline against which future improvements can be measured.

However, the full extent of the problem has not been well documented. No systematic assessment of nitrate concentrations across the GWMA has been performed, complicating the interpretation of localized monitoring efforts that may indicate nitrate from distant or local sources, and making long-term trend analysis difficult.

Groundwater in many areas of the GWMA has not been sampled for nitrate, creating gaps in the understanding of nitrate concentrations in aquifers tapped for drinking water. The nitrate data that do exist were not necessarily collected for the purpose of comparison to previous nitrate concentrations to determine if levels are increasing or decreasing over long time scales. The existing data also were not collected with the goal of determining the seasonal variation of nitrate concentrations in groundwater, which is likely to indicate agricultural practices such as tillage, fertilization, and irrigation.

The purpose of this data collection effort is to collect sufficient nitrate concentration data from the lower Yakima River Basin GWMA to fill identified critical gaps in the current groundwater nitrate inventory. The data collected will assist in documenting the GWMA's current nitrate conditions and provide a baseline against which future nitrate assessments can be compared, and will provide insight into the design and sampling frequency of a long-term monitoring program. This report describes the results of this data collection effort. 


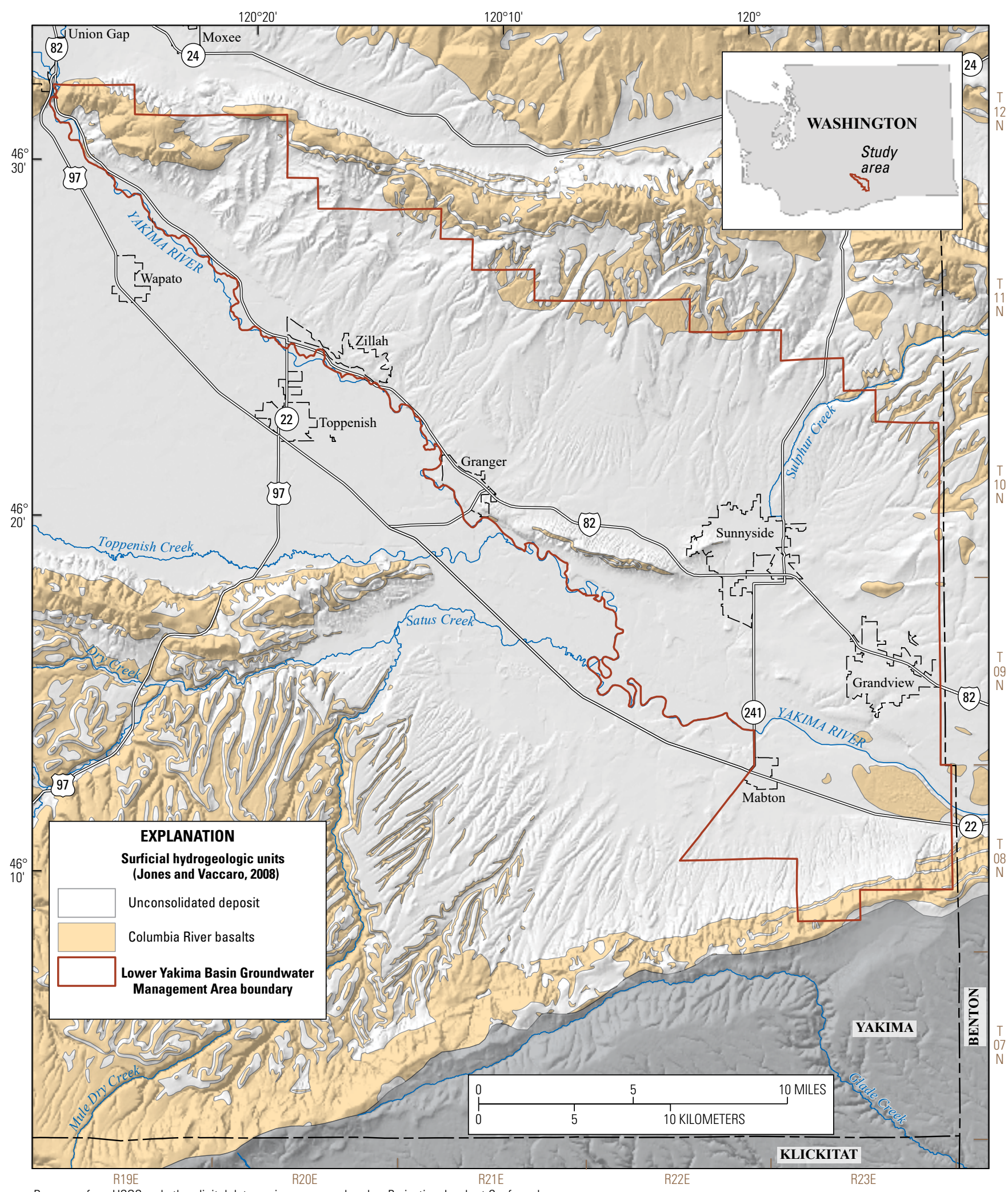

Base map from USGS and other digital data, various years and scales. Projection: Lambert Conformal Conic, State Plane Washington South. Horizontal datum is North American Datum of 1983

Figure 1. Location of the Groundwater Management Area and surficial hydrogeologic units, lower Yakima River Basin, Yakima County, Washington. 


\section{Description of Study Area}

The Yakima River Basin extends from the Cascade Mountains east to the Columbia River. Streamflow averages 4.1 million acre-feet per year (acre-ft/yr), and is intensively managed using five storage reservoirs and irrigation diversions of about 2.8 million acre-ft/yr (Ely and others, 2011). Eastwest trending ridges separate the Yakima River Basin into several broad valleys (subbasins) that slope gently toward the Yakima River, and contain most of the population and economic activity in the basin. The Toppenish/Benton subbasin, the largest and most southerly of the five subbasins, encompasses almost 1,500 $\mathrm{mi}^{2}$ between Ahtanum Ridge and Rattlesnake Hills on the north and Toppenish Ridge and the Horse Heaven Hills on the south. The lower Yakima River Basin GWMA (fig. 1) is within this subbasin.

The Toppenish/Benton subbasin is largely filled with deposits of alluvial fan, loess, dune sand, alluvial terrace, continental sedimentary, and Touchet Formation and Ellensburg Formation deposits. These sediments are more than 1,200 -ft thick west of the town of Toppenish and gradually thin to zero thickness at the northern end of the subbasin where the underlying basalt outcrops. The unconsolidated sediments remain about 1,000-ft thick south of the Yakima River (and the GWMA) until the basin abruptly ends against the steep Toppenish Ridge (Jones and others, 2006).

Irrigated cropland is the major land use activity within the subbasin, encompassing 70-80 percent of the area. The primary crop types within the subbasin include orchards, vegetables, hay, mint, and hops (Washington State Department of Ecology, 2010). Dairy and animal feeding operations are also prevalent within the subbasin near the cities of Sunnyside, Granger, Mabton, and Grandview (Washington State Department of Ecology, 2010). Substantial amounts of nitrogen have been applied to the land surface in the lower Yakima River Basin for decades, primarily as fertilizer for row crops, but also in recent years as animal waste (manure) (Washington State Department of Ecology, 2010).

About one-third of the basin population is not served by public supply systems and instead relies on private wells (Washington State Department of Ecology, 2010). Residential drinking-water wells in the GWMA are typically shallow, with some as shallow as 10-ft deep. Wells that typically require a high flow rate are more likely to be drilled through the entire thickness of basin sediments to penetrate the Wanapum or Saddle Mountain basalts that underlie the subbasin.

\section{Sample Collection Design and Methods}

\section{Sites Selection}

Groundwater samples were collected from 156 existing wells (table 1). Well selection was based on the following criteria: known and documented location and completion depth or open interval (drillers' log), the ability to collect a sample prior to any treatment or filtration, and spatial distribution across the GWMA (table 1, fig. 2). Candidate domestic wells for this study consisted of those listed in the USGS National Water Information System database, Washington State Department of Ecology well log database, and from a well assessment survey conducted by Yakima County in 2015 and 2016. Further screening of candidate wells took place in the field, based on owner permission and ability to collect a representative sample. Groundwater samples were collected about every 6 weeks (a total of 6 times) from April to December 2017.

Surface water, in agricultural drains, was sampled from 24 sites, spatially distributed across the GWMA (table 2, fig. 2). Drain sites were pre-selected by the GWMA. Suitability of a site was determined during field reconnaissance based on the ability to gain access to the site, and the ability to obtain a representative grab (dip) sample. Each site was sampled about once every 6 weeks for a total of 7 times between April and December 2017.

\section{Sample Collection}

All sample collection, processing, and field analyses were in accordance with applicable USGS procedures as described in the National Field Manual (U.S. Geological Survey, variously dated). Groundwater samples were collected prior to any filtration or treatment, directly from a spigot after a sufficient well casing volume of water was purged from the well and after allowing field measurements of $\mathrm{pH}$, specific conductance, and dissolved oxygen to stabilize to within $0.1 \mathrm{pH}$ unit, 3 percent, and $0.3 \mathrm{mg} / \mathrm{L}$, respectively. Because of the limitations of wading the drains during the irrigation season, and their uniform shape and well-mixed characteristics, a grab (dip) sample was collected in the centroid of flow along with field measurements of $\mathrm{pH}$, specific conductance, and dissolved oxygen. 
Table 1. Location and description of groundwater wells sampled for nitrate in the Groundwater Management Area, Yakima County, Washington, April-December 2017.

[Well No: See section, "Well Numbering System" in front of report for naming convention explanation. Latitude and Longitude: Latitude and longitude at well in decimal degrees referenced to North American Datum of 1983. Land surface elevation: in feet above North American Vertical Datum of 1988. Type of opening: P, perforated; X, open hole; O, open end; S, slotted screen. Type of log available: D, Driller. Abbreviation: ft BLS, foot below land surface; -, no data available]

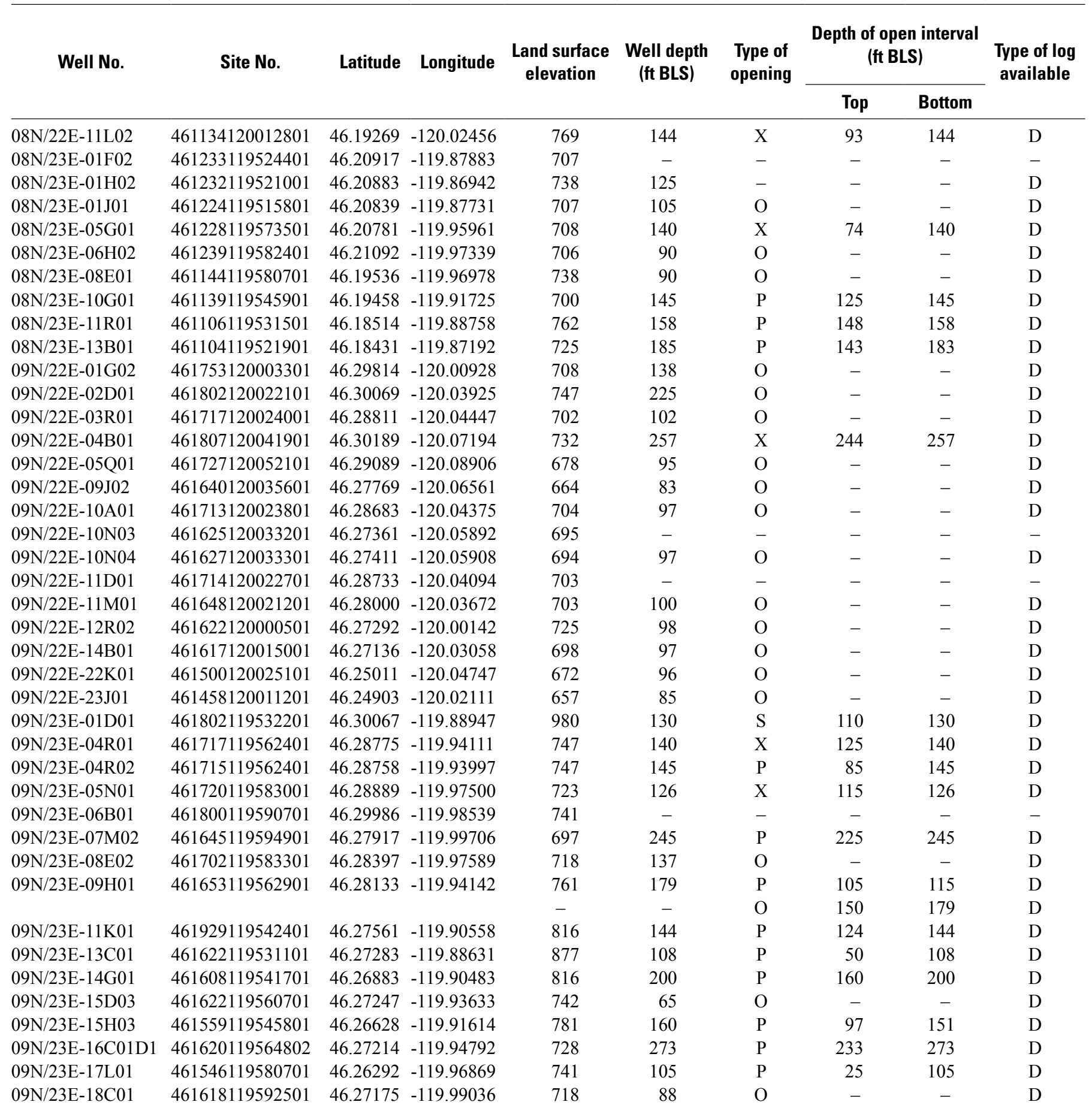


Table 1. Location and description of groundwater wells sampled for nitrate in the Groundwater Management Area, Yakima County, Washington, April-December 2017.-Continued

\begin{tabular}{|c|c|c|c|c|c|c|c|c|c|}
\hline \multirow[t]{2}{*}{ Well No. } & \multirow[t]{2}{*}{ Site No. } & \multirow[t]{2}{*}{ Latitude } & \multirow[t]{2}{*}{ Longitude } & \multirow{2}{*}{$\begin{array}{l}\text { Land surface } \\
\text { elevation }\end{array}$} & \multirow{2}{*}{$\begin{array}{l}\text { Well depth } \\
\text { (ft BLS) }\end{array}$} & \multirow{2}{*}{$\begin{array}{l}\text { Type of } \\
\text { opening }\end{array}$} & \multicolumn{2}{|c|}{$\begin{array}{c}\text { Depth of open interval } \\
\text { (ft BLS) }\end{array}$} & \multirow{2}{*}{$\begin{array}{l}\text { Type of log } \\
\text { available }\end{array}$} \\
\hline & & & & & & & Top & Bottom & \\
\hline 09N/23E-19D03 & 461521119595101 & 46.25578 & -119.99850 & 674 & 96 & $\mathrm{O}$ & - & - & $\mathrm{D}$ \\
\hline 09N/23E-19Q01 & 461439119590401 & 46.24406 & -119.98439 & 672 & 122 & $\mathrm{O}$ & - & - & $\mathrm{D}$ \\
\hline 09N/23E-20A01 & 461529119574701 & 46.25817 & -119.96306 & 750 & 100 & $\mathrm{P}$ & 80 & 100 & $\mathrm{D}$ \\
\hline 09N/23E-21P01 & 461446119565501 & 46.24611 & -119.94858 & 796 & 145 & $\mathrm{P}$ & 125 & 145 & $\mathrm{D}$ \\
\hline 09N/23E-24L01 & 461456119530501 & 46.24883 & -119.88478 & 822 & 125 & $\mathrm{P}$ & 85 & 125 & $\mathrm{D}$ \\
\hline 09N/23E-25J01 & 461402119522701 & 46.23403 & -119.87417 & 819 & 85 & $\mathrm{P}$ & 45 & 85 & $\mathrm{D}$ \\
\hline 09N/23E-26B01 & 461432119541701 & 46.24231 & -119.90481 & 830 & 190 & $\mathrm{P}$ & 170 & 190 & $\mathrm{D}$ \\
\hline $09 \mathrm{~N} / 23 \mathrm{E}-27 \mathrm{~B} 02$ & 461435119553201 & 46.24303 & -119.92556 & 806 & 145 & $\mathrm{X}$ & 116 & 145 & $\mathrm{D}$ \\
\hline 09N/23E-28G01 & 461414119564501 & 46.23711 & -119.94572 & 741 & 160 & $\mathrm{X}$ & 117 & 160 & $\mathrm{D}$ \\
\hline 09N/23E-29B02 & 461424119574801 & 46.24011 & -119.96319 & 688 & 180 & $\mathrm{P}$ & 140 & 180 & $\mathrm{D}$ \\
\hline 09N/23E-31K01 & 461312119592001 & 46.21994 & -119.98894 & 706 & 142 & $\mathrm{O}$ & - & - & $\mathrm{D}$ \\
\hline 09N/23E-34M01 & 461316119560801 & 46.22108 & -119.93547 & 670 & 100 & $\mathrm{O}$ & - & - & $\mathrm{D}$ \\
\hline 09N/23E-35K01 & 461307119540201 & 46.21850 & -119.90058 & 668 & 98 & $\mathrm{O}$ & - & - & $\mathrm{D}$ \\
\hline 09N/23E-36J01 & 461316119523701 & 46.22097 & -119.87706 & 771 & 120 & $\mathrm{P}$ & 80 & 120 & $\mathrm{D}$ \\
\hline $10 \mathrm{~N} / 21 \mathrm{E}-01 \mathrm{G} 01$ & 462258120080701 & 46.38289 & -120.13533 & 885 & 102 & $\mathrm{O}$ & - & - & $\mathrm{D}$ \\
\hline $10 \mathrm{~N} / 21 \mathrm{E}-02 \mathrm{~N} 01$ & 462234120095501 & 46.37614 & -120.16517 & 851 & 154 & $\mathrm{O}$ & - & - & $\mathrm{D}$ \\
\hline 10N/21E-03D02 & 462314120111801 & 46.38708 & -120.18833 & 900 & 198 & $\mathrm{O}$ & - & - & $\mathrm{D}$ \\
\hline 10N/21E-04P02 & 462232120120801 & 46.37533 & -120.20333 & 824 & 196 & $\mathrm{O}$ & - & - & $\mathrm{D}$ \\
\hline $10 \mathrm{~N} / 21 \mathrm{E}-05 \mathrm{~A} 01$ & 462321120123901 & 46.38900 & -120.21206 & 843 & 218 & $\mathrm{O}$ & - & - & $\mathrm{D}$ \\
\hline 10N/21E-09F01 & 462206120115701 & 46.36842 & -120.19914 & 785 & 127 & $\mathrm{~S}$ & 117 & 127 & $\mathrm{D}$ \\
\hline $10 \mathrm{~N} / 21 \mathrm{E}-11 \mathrm{M} 01$ & 462206120100201 & 46.36822 & -120.16719 & 794 & 157 & $\mathrm{O}$ & - & - & $\mathrm{D}$ \\
\hline 10N/21E-12R01 & 462147120073501 & 46.36303 & -120.12628 & 821 & - & - & - & - & - \\
\hline $10 \mathrm{~N} / 21 \mathrm{E}-13 \mathrm{~N} 01$ & 462047120083301 & 46.34644 & -120.14242 & 784 & 221 & $\mathrm{O}$ & - & - & $\mathrm{D}$ \\
\hline 10N/21E-15E01 & 462114120105701 & 46.35497 & -120.18083 & 784 & 167 & $\mathrm{O}$ & - & - & $\mathrm{D}$ \\
\hline $10 \mathrm{~N} / 21 \mathrm{E}-16 \mathrm{~B} 01$ & 462137120115001 & 46.36019 & -120.19733 & 764 & 65 & $\mathrm{X}$ & 60 & 65 & D \\
\hline $10 \mathrm{~N} / 21 \mathrm{E}-16 \mathrm{G} 02$ & 462126120114501 & 46.35694 & -120.19689 & 763 & 100 & $\mathrm{O}$ & - & - & $\mathrm{D}$ \\
\hline $10 \mathrm{~N} / 21 \mathrm{E}-23 \mathrm{~A} 01$ & 462047120084501 & 46.34631 & -120.14717 & 766 & 240 & $\mathrm{O}$ & - & - & $\mathrm{D}$ \\
\hline 10N/21E-24J01 & 462010120072901 & 46.33886 & -120.12572 & 778 & 200 & $\mathrm{O}$ & - & - & $\mathrm{D}$ \\
\hline $10 \mathrm{~N} / 22 \mathrm{E}-01 \mathrm{~F} 01$ & 462305120003901 & 46.38456 & -120.01203 & 1,067 & 250 & $\mathrm{X}$ & 193 & 250 & $\mathrm{D}$ \\
\hline $10 \mathrm{~N} / 22 \mathrm{E}-03 \mathrm{~B} 02$ & 462323120030001 & 46.38903 & -120.05119 & 1,003 & 120 & $\mathrm{O}$ & - & - & $\mathrm{D}$ \\
\hline 10N/22E-04J01 & 462254120034301 & 46.38136 & -120.06300 & 1,015 & 226 & $\mathrm{O}$ & - & - & $\mathrm{D}$ \\
\hline $10 \mathrm{~N} / 22 \mathrm{E}-05 \mathrm{P} 01$ & 462231120054001 & 46.37519 & -120.09594 & 919 & 205 & $\mathrm{X}$ & 185 & 205 & $\mathrm{D}$ \\
\hline $10 \mathrm{~N} / 22 \mathrm{E}-06 \mathrm{~A} 01$ & 462321120062801 & 46.38914 & -120.10786 & 994 & 227 & $P$ & 217 & 227 & $\mathrm{D}$ \\
\hline $10 \mathrm{~N} / 22 \mathrm{E}-07 \mathrm{~N} 01$ & 462145120072701 & 46.36239 & -120.12528 & 808 & 178 & $\mathrm{O}$ & - & - & $\mathrm{D}$ \\
\hline $10 \mathrm{~N} / 22 \mathrm{E}-08 \mathrm{~F} 02$ & 462206120054001 & 46.36836 & -120.09458 & 874 & 162 & $\mathrm{O}$ & - & - & $\mathrm{D}$ \\
\hline $10 \mathrm{~N} / 22 \mathrm{E}-08 \mathrm{H} 01$ & 462203120050001 & 46.36761 & -120.08328 & 867 & 142 & $\mathrm{O}$ & - & - & $\mathrm{D}$ \\
\hline $10 \mathrm{~N} / 22 \mathrm{E}-08 \mathrm{~K} 04$ & 462152120052401 & 46.36447 & -120.09000 & 855 & 171 & $\mathrm{O}$ & - & - & $\mathrm{D}$ \\
\hline 10N/22E-08L01 & 462203120054001 & 46.36758 & -120.09431 & 873 & 161 & $\mathrm{O}$ & - & - & $\mathrm{D}$ \\
\hline $10 \mathrm{~N} / 22 \mathrm{E}-11 \mathrm{~J} 02$ & 462202120011401 & 46.36708 & -120.02044 & 908 & 175 & $\mathrm{X}$ & 160 & 175 & $\mathrm{D}$ \\
\hline $10 \mathrm{~N} / 22 \mathrm{E}-13 \mathrm{E} 02$ & 462115120011001 & 46.35425 & -120.01947 & 858 & 255 & $\mathrm{P}$ & 235 & 255 & $\mathrm{D}$ \\
\hline $10 \mathrm{~N} / 22 \mathrm{E}-14 \mathrm{~K} 01$ & 462105120014501 & 46.35136 & -120.02911 & 813 & 205 & $\mathrm{P}$ & 185 & 205 & $\mathrm{D}$ \\
\hline $10 \mathrm{~N} / 22 \mathrm{E}-17 \mathrm{C} 02$ & 462136120054601 & 46.36011 & -120.09611 & 838 & - & - & - & - & - \\
\hline $10 \mathrm{~N} / 22 \mathrm{E}-18 \mathrm{G} 03$ & 462122120063801 & 46.35622 & -120.11044 & 815 & 161 & $\mathrm{O}$ & - & - & D \\
\hline 10N/22E-19L01 & 462019120070401 & 46.33867 & -120.11789 & 783 & 237 & $\mathrm{O}$ & - & - & $\mathrm{D}$ \\
\hline $10 \mathrm{~N} / 22 \mathrm{E}-20 \mathrm{~N} 02$ & 461956120060401 & 46.33231 & -120.10117 & 774 & 202 & $\mathrm{P}$ & 181 & 202 & $\mathrm{D}$ \\
\hline $10 \mathrm{~N} / 22 \mathrm{E}-21 \mathrm{R} 02$ & 461955120035601 & 46.33186 & -120.06569 & 778 & 243 & $\mathrm{O}$ & - & - & $\mathrm{D}$ \\
\hline
\end{tabular}


Table 1. Location and description of groundwater wells sampled for nitrate in the Groundwater Management Area, Yakima County, Washington, April-December 2017.-Continued

\begin{tabular}{|c|c|c|c|c|c|c|c|c|c|}
\hline \multirow[t]{2}{*}{ Well No. } & \multirow[t]{2}{*}{ Site No. } & \multirow[t]{2}{*}{ Latitude } & \multirow[t]{2}{*}{ Longitude } & \multirow{2}{*}{$\begin{array}{l}\text { Land surface } \\
\text { elevation }\end{array}$} & \multirow{2}{*}{$\begin{array}{l}\text { Well depth } \\
\text { (ft BLS) }\end{array}$} & \multirow{2}{*}{$\begin{array}{l}\text { Type of } \\
\text { opening }\end{array}$} & \multicolumn{2}{|c|}{$\begin{array}{c}\text { Depth of open interval } \\
\text { (ft BLS) }\end{array}$} & \multirow{2}{*}{$\begin{array}{l}\text { Type of log } \\
\text { available }\end{array}$} \\
\hline & & & & & & & Top & Bottom & \\
\hline $10 \mathrm{~N} / 22 \mathrm{E}-22 \mathrm{P} 01$ & 462012120031001 & 46.33253 & -120.05178 & 774 & 60 & $\mathrm{O}$ & - & - & $\mathrm{D}$ \\
\hline $10 \mathrm{~N} / 22 \mathrm{E}-23 \mathrm{H} 02$ & 462019120011801 & 46.33872 & -120.02181 & 782 & 213 & $\mathrm{O}$ & - & - & $\mathrm{D}$ \\
\hline $10 \mathrm{~N} / 22 \mathrm{E}-24 \mathrm{M} 01$ & 462014120010001 & 46.33728 & -120.01681 & 799 & 238 & $\mathrm{O}$ & - & - & $\mathrm{D}$ \\
\hline $10 \mathrm{~N} / 22 \mathrm{E}-27 \mathrm{~N} 01$ & 461902120033401 & 46.31696 & -120.06063 & 803 & 135 & $\mathrm{X}$ & 122 & 135 & $\mathrm{D}$ \\
\hline $10 \mathrm{~N} / 22 \mathrm{E}-29 \mathrm{D} 01$ & 461952120055701 & 46.33119 & -120.09911 & 771 & 79 & $\mathrm{O}$ & - & - & $\mathrm{D}$ \\
\hline $10 \mathrm{~N} / 22 \mathrm{E}-30 \mathrm{~B} 01$ & 461947120063901 & 46.32853 & -120.11275 & 772 & 290 & $\mathrm{O}$ & - & - & $\mathrm{D}$ \\
\hline $10 \mathrm{~N} / 22 \mathrm{E}-34 \mathrm{~B} 01$ & 461848120024801 & 46.31328 & -120.04656 & 893 & 98 & $\mathrm{O}$ & - & - & $\mathrm{D}$ \\
\hline $10 \mathrm{~N} / 22 \mathrm{E}-34 \mathrm{~B} 02$ & 461858120030301 & 46.31606 & -120.05078 & 801 & 227 & $\mathrm{X}$ & 222 & 227 & $\mathrm{D}$ \\
\hline $10 \mathrm{~N} / 23 \mathrm{E}-22 \mathrm{~L} 01$ & 462008119553601 & 46.33567 & -119.92669 & 946 & 193 & $\mathrm{~S}$ & 173 & 193 & $\mathrm{D}$ \\
\hline $10 \mathrm{~N} / 23 \mathrm{E}-23 \mathrm{R} 01$ & 462006119535701 & 46.33486 & -119.89928 & 1,025 & 100 & $\mathrm{X}$ & 80 & 100 & $\mathrm{D}$ \\
\hline $10 \mathrm{~N} / 23 \mathrm{E}-25 \mathrm{~J} 07$ & 461916119524001 & 46.32122 & -119.87772 & 1,225 & 399 & $\mathrm{~S}$ & 372 & 392 & $\mathrm{D}$ \\
\hline 10N/23E-27N01 & 461903119561001 & 46.31744 & -119.93608 & 844 & 185 & $\mathrm{P}$ & 135 & 165 & $\mathrm{D}$ \\
\hline $10 \mathrm{~N} / 23 \mathrm{E}-28 \mathrm{~F} 01$ & 461929119570201 & 46.32483 & -119.95053 & 807 & 245 & $\mathrm{P}$ & 225 & 245 & $\mathrm{D}$ \\
\hline $10 \mathrm{~N} / 23 \mathrm{E}-29 \mathrm{~A} 01$ & 461947119573401 & 46.32958 & -119.96060 & 783 & 52 & $\mathrm{O}$ & - & - & $\mathrm{D}$ \\
\hline $10 \mathrm{~N} / 23 \mathrm{E}-30 \mathrm{~A} 01$ & 461952119590201 & 46.33122 & -119.98400 & 765 & 70 & $\mathrm{O}$ & - & - & $\mathrm{D}$ \\
\hline $10 \mathrm{~N} / 23 \mathrm{E}-31 \mathrm{E} 02$ & 461846119594901 & 46.31275 & -119.99692 & 731 & 115 & $\mathrm{X}$ & 102 & 115 & $\mathrm{D}$ \\
\hline $10 \mathrm{~N} / 23 \mathrm{E}-32 \mathrm{~K} 02$ & 461827119580201 & 46.30758 & -119.96711 & 745 & 169 & $\mathrm{O}$ & - & - & $\mathrm{D}$ \\
\hline $10 \mathrm{~N} / 23 \mathrm{E}-33 \mathrm{D} 01$ & 461900119571901 & 46.31683 & -119.95522 & 788 & 63 & $\mathrm{X}$ & 58 & 63 & $\mathrm{D}$ \\
\hline $10 \mathrm{~N} / 23 \mathrm{E}-34 \mathrm{~A} 01$ & 461859119550801 & 46.31631 & -119.91883 & 905 & 115 & $\mathrm{~S}$ & 73 & 93 & $\mathrm{D}$ \\
\hline $11 \mathrm{~N} / 20 \mathrm{E}-09 \mathrm{~L} 02$ & 462705120193501 & 46.45131 & -120.32647 & 931 & 162 & $\mathrm{X}$ & 117 & 162 & $\mathrm{D}$ \\
\hline $11 \mathrm{~N} / 20 \mathrm{E}-10 \mathrm{C} 02$ & 462742120182701 & 46.46181 & -120.30753 & 1,207 & 340 & $X$ & 220 & 340 & $\mathrm{D}$ \\
\hline $11 \mathrm{~N} / 20 \mathrm{E}-10 \mathrm{P} 01$ & 462653120182401 & 46.44792 & -120.30664 & 973 & 250 & $\mathrm{P}$ & 230 & 250 & $\mathrm{D}$ \\
\hline $11 \mathrm{~N} / 20 \mathrm{E}-11 \mathrm{R} 01$ & 462651120162101 & 46.44758 & -120.27247 & 1,075 & 280 & $\mathrm{X}$ & 272 & 280 & $\mathrm{D}$ \\
\hline $11 \mathrm{~N} / 20 \mathrm{E}-12 \mathrm{P} 02$ & 462653120155901 & 46.44803 & -120.26647 & 1,140 & 382 & $\mathrm{X}$ & 360 & 382 & $\mathrm{D}$ \\
\hline 11N/20E-13J01 & 462624120150901 & 46.44003 & -120.25242 & 1,011 & 170 & $\mathrm{P}$ & 130 & 150 & $\mathrm{D}$ \\
\hline $11 \mathrm{~N} / 20 \mathrm{E}-14 \mathrm{M} 03$ & 462616120173101 & 46.43767 & -120.29203 & 928 & 145 & $\mathrm{P}$ & 125 & 145 & $\mathrm{D}$ \\
\hline $11 \mathrm{~N} / 20 \mathrm{E}-15 \mathrm{~B} 02$ & 462640120180901 & 46.44453 & -120.30258 & 928 & 195 & $\mathrm{X}$ & 193 & 195 & $\mathrm{D}$ \\
\hline $11 \mathrm{~N} / 20 \mathrm{E}-21 \mathrm{~B} 02$ & 462549120192801 & 46.43033 & -120.32431 & 794 & 67 & $\mathrm{O}$ & - & - & $\mathrm{D}$ \\
\hline $11 \mathrm{~N} / 20 \mathrm{E}-22 \mathrm{Q} 01$ & 462511120175701 & 46.41983 & -120.29917 & 828 & 125 & $\mathrm{P}$ & 105 & 125 & $\mathrm{D}$ \\
\hline $11 \mathrm{~N} / 20 \mathrm{E}-23 \mathrm{Q} 02$ & 462508120164301 & 46.41886 & -120.27872 & 854 & 138 & $\mathrm{O}$ & - & - & $\mathrm{D}$ \\
\hline $11 \mathrm{~N} / 20 \mathrm{E}-24 \mathrm{E} 02$ & 462534120162001 & 46.42600 & -120.27214 & 882 & 210 & $\mathrm{P}$ & 190 & 210 & $\mathrm{D}$ \\
\hline $11 \mathrm{~N} / 20 \mathrm{E}-24 \mathrm{~J} 03$ & 462528120151101 & 46.42450 & -120.25294 & 949 & 238 & $\mathrm{O}$ & - & - & $\mathrm{D}$ \\
\hline $11 \mathrm{~N} / 20 \mathrm{E}-24 \mathrm{~N} 01$ & 462508120160301 & 46.41886 & -120.26739 & 884 & 140 & $\mathrm{X}$ & 133 & 140 & $\mathrm{D}$ \\
\hline $11 \mathrm{~N} / 20 \mathrm{E}-24 \mathrm{P} 03$ & 462519120155601 & 46.42186 & -120.26547 & 890 & 152 & $\mathrm{P}$ & 125 & 152 & $\mathrm{D}$ \\
\hline $11 \mathrm{~N} / 20 \mathrm{E}-24 \mathrm{R} 01$ & 462511120152201 & 46.41972 & -120.25619 & 889 & 275 & $\mathrm{O}$ & - & - & $\mathrm{D}$ \\
\hline
\end{tabular}


Table 1. Location and description of groundwater wells sampled for nitrate in the Groundwater Management Area, Yakima County, Washington, April-December 2017.-Continued

\begin{tabular}{|c|c|c|c|c|c|c|c|c|c|}
\hline \multirow{2}{*}{ Well No. } & \multirow{2}{*}{ Site No. } & \multirow{2}{*}{ Latitude } & \multirow[t]{2}{*}{ Longitude } & \multirow{2}{*}{$\begin{array}{l}\text { Land surface } \\
\text { elevation }\end{array}$} & \multirow{2}{*}{$\begin{array}{l}\text { Well depth } \\
\text { (ft BLS) }\end{array}$} & \multirow{2}{*}{$\begin{array}{c}\text { Type of } \\
\text { opening }\end{array}$} & \multicolumn{2}{|c|}{$\begin{array}{c}\text { Depth of open interval } \\
\text { (ft BLS) }\end{array}$} & \multirow{2}{*}{$\begin{array}{l}\text { Type of log } \\
\text { available }\end{array}$} \\
\hline & & & & & & & Top & Bottom & \\
\hline $11 \mathrm{~N} / 20 \mathrm{E}-25 \mathrm{~L} 01$ & 462437120154601 & 46.41017 & -120.26264 & 856 & 165 & $\mathrm{O}$ & - & - & $\mathrm{D}$ \\
\hline $11 \mathrm{~N} / 20 \mathrm{E}-26 \mathrm{~F} 01$ & 462440120170401 & 46.41122 & -120.28433 & 820 & 135 & $\mathrm{O}$ & - & - & $\mathrm{D}$ \\
\hline 11N/21E-06R01D1 & 462745120135701 & 46.46244 & -120.23256 & 1,241 & 663 & $\mathrm{P}$ & 603 & 663 & $\mathrm{D}$ \\
\hline 11N/21E-18G01 & 462637120142301 & 46.44372 & -120.23969 & 1,124 & 325 & $\mathrm{~S}$ & 305 & 325 & D \\
\hline 11N/21E-19J01 & 462523120135001 & 46.42297 & -120.23042 & 1,002 & 260 & $\mathrm{P}$ & 210 & 250 & D \\
\hline \multirow[t]{2}{*}{$11 \mathrm{~N} / 21 \mathrm{E}-20 \mathrm{~N} 01$} & 462508120133301 & 46.41897 & -120.22575 & 1,044 & 795 & $\mathrm{P}$ & 453 & 510 & $\mathrm{D}$ \\
\hline & & & & & & & 610 & 658 & $\mathrm{D}$ \\
\hline $11 \mathrm{~N} / 21 \mathrm{E}-21 \mathrm{~N} 01$ & 462509120123201 & 46.41917 & -120.20894 & 1,047 & 208 & $\mathrm{O}$ & - & - & D \\
\hline $11 \mathrm{~N} / 21 \mathrm{E}-21 \mathrm{~N} 02$ & 462515120123101 & 46.42072 & -120.20850 & 1,066 & 281 & $\mathrm{X}$ & 278 & 281 & D \\
\hline $11 \mathrm{~N} / 21 \mathrm{E}-27 \mathrm{~A} 01$ & 462504120100301 & 46.41769 & -120.16761 & 1,135 & 376 & $\mathrm{O}$ & 301 & 376 & $\mathrm{D}$ \\
\hline $11 \mathrm{~N} / 21 \mathrm{E}-28 \mathrm{H} 01$ & 462453120112301 & 46.41472 & -120.18981 & 1,043 & 281 & $\mathrm{P}$ & 260 & 280 & $\mathrm{D}$ \\
\hline 11N/21E-29M05 & 462435120134401 & 46.40983 & -120.22892 & 923 & 198 & $\mathrm{O}$ & - & - & $\mathrm{D}$ \\
\hline $11 \mathrm{~N} / 21 \mathrm{E}-30 \mathrm{~F} 03$ & 462443120143601 & 46.41169 & -120.24439 & 933 & 220 & $\mathrm{X}$ & 214 & 220 & $\mathrm{D}$ \\
\hline $11 \mathrm{~N} / 21 \mathrm{E}-31 \mathrm{D} 01$ & 462408120145701 & 46.40211 & -120.24908 & 887 & - & - & - & - & - \\
\hline $11 \mathrm{~N} / 21 \mathrm{E}-32 \mathrm{~N} 01$ & 462324120133801 & 46.39003 & -120.22708 & 839 & 199 & $\mathrm{O}$ & - & - & $\mathrm{D}$ \\
\hline $11 \mathrm{~N} / 21 \mathrm{E}-33 \mathrm{C} 02$ & 462411120121401 & 46.40303 & -120.20394 & 904 & 158 & $\mathrm{O}$ & - & - & $\mathrm{D}$ \\
\hline $11 \mathrm{~N} / 21 \mathrm{E}-33 \mathrm{M} 01$ & 462338120122201 & 46.39386 & -120.20608 & 876 & 354 & $\mathrm{O}$ & - & - & $\mathrm{D}$ \\
\hline 12N/19E-27Q01 & 462936120253601 & 46.49325 & -120.42672 & 902 & 120 & $P$ & 100 & 120 & $\mathrm{D}$ \\
\hline $12 \mathrm{~N} / 19 \mathrm{E}-35 \mathrm{E} 01$ & 462915120245201 & 46.48744 & -120.41447 & 882 & 141 & $\mathrm{X}$ & 138 & 141 & $\mathrm{D}$ \\
\hline $12 \mathrm{~N} / 19 \mathrm{E}-36 \mathrm{D} 01$ & 462928120234201 & 46.49125 & -120.39500 & 1,091 & 405 & $\mathrm{P}$ & 365 & 405 & $\mathrm{D}$ \\
\hline $12 \mathrm{~N} / 20 \mathrm{E}-31 \mathrm{~B} 02$ & 462922120214101 & 46.49083 & -120.35789 & 1,060 & 402 & $\mathrm{~S}$ & 330 & 400 & D \\
\hline $12 \mathrm{~N} / 20 \mathrm{E}-33 \mathrm{Q} 02$ & 462837120191101 & 46.47689 & -120.31964 & 1,155 & 322 & $\mathrm{P}$ & 302 & 322 & $\mathrm{D}$ \\
\hline
\end{tabular}

Field measurements were monitored using a Yellow Springs Instruments $\left(\mathrm{YSI}^{\circledR}\right)$ 600XLM or $6920 \mathrm{~V} 2$ data sonde. The sonde was calibrated prior to use, as described in the National Field Manual (U.S. Geological Survey, variously dated). The specific conductance sensor was calibrated daily with standard reference solutions $(1,000 \mu \mathrm{S} / \mathrm{cm}$ and checked with solutions ranging from 250 to $750 \mu \mathrm{S} / \mathrm{cm}$ ); the $\mathrm{pH}$ sensor was calibrated daily with two $\mathrm{pH}$ standards (7 and 10, and checked against $\mathrm{pH} 4$ buffer solution); and the dissolved oxygen (DO) sensor was calibrated daily using the air-saturated water method and occasionally verified with zero-DO solution.

Samples for analysis of nitrate concentrations were filtered, in the field, through a $0.45 \mu \mathrm{m}$ membrane syringefilter into polyethylene bottles, chilled, and sent to the USGS National Water Quality Laboratory (NWQL) in Lakewood, Colorado.
In addition to collecting environmental samples, qualitycontrol samples consisting of blank and replicate samples, made up 8 percent of all well samples taken (table 3 ). Blank samples are contaminant-free water submitted to the laboratory and analyzed to ensure that environmental samples have not been contaminated. Two types of blanks were prepared, an equipment blank, prepared by transferring blank water through laboratory cleaned sampling equipment to ensure that neither is a source of contamination; and a field blank, prepared concurrently with environmental samples to ensure that field and laboratory procedures do not result in contaminant or carry-over between sites. Replicates are duplicate environmental samples from a site submitted to the laboratory to determine whether field procedures and analytical results are reproducible. 


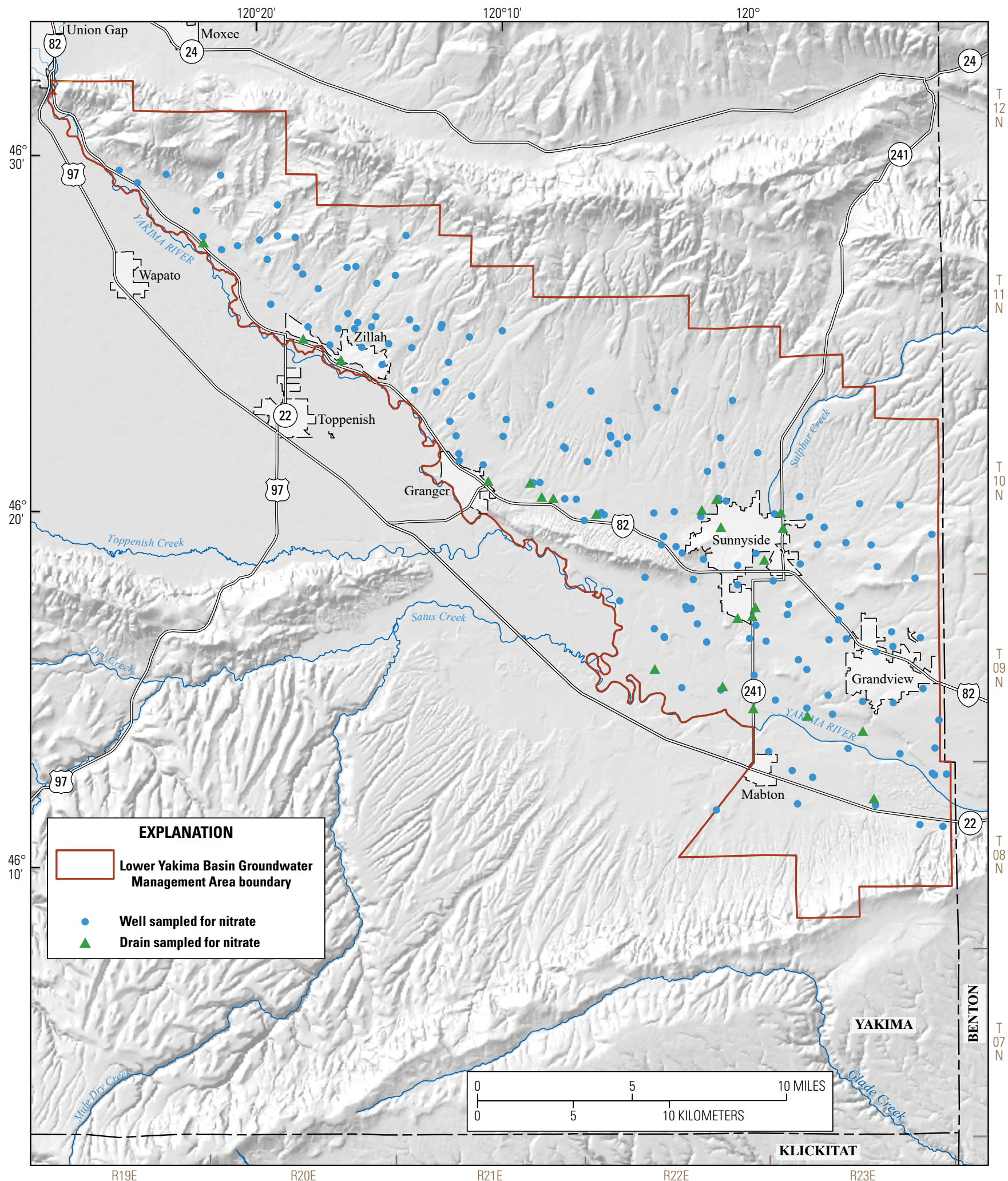

Base map from USGS and other digital data, various years and scales. Projection: Lambert Conformal Conic, State Plane Washington South. Horizontal datum is North American Datum of 1983

Figure 2. Wells and drains sampled for nitrate in the lower Yakima River Basin, Groundwater Management Area, Yakima County, Washington, April-December 2017. 
Table 2. Location of drains sampled for nitrate in the lower Yakima River Basin, Groundwater Management Area, Yakima County, Washington, April-December 2017.

[Latitude and Longitude: Latitude and longitude at site in decimal degrees referenced to North American Datum of 1983]

\begin{tabular}{llll}
\hline \multicolumn{1}{c}{ Station name } & \multicolumn{1}{c}{ Site No. } & Latitude & Longitude \\
\hline Buena drain at westbound I-82 near Buena, WA & 12505315 & 46.4142 & -120.3025 \\
DID 18 Drain at Sunnyside, WA & 12508790 & 46.3246 & -119.9784 \\
DID 3 Drain near Sunnyside, WA & 12508840 & 46.2826 & -120.0095 \\
DID 7 Drain near Mabton, WA & 12508670 & 46.2589 & -120.0656 \\
DR2 NEET Site Number 3 & 462021120075700 & 46.3390 & -120.1337 \\
Drain 31 at West Charvet Road at Mabton, WA & 12508988 & 46.2401 & -119.9993 \\
Drain 35 off Charvet Road, near Grandview, WA & 12508905 & 46.2365 & -119.9629 \\
Grandview Drain at Chase Road near Grandview, WA & 12508997 & -119.9256 \\
Granger Drain at Granger, WA & 12505450 & 46.2293 & -120.1870 \\
Joint drain 27.5 at Vanbelle Road & 462047120085300 & 46.3463 & -120.1492 \\
Joint drain 34.2 at Woodin Road & 462018120012000 & 46.3382 & -120.0232 \\
Joint drain 43.9 at Mabton Sunnyside Road & 461700119595400 & 46.2833 & -119.9994 \\
Joint drain 1 at Bus Road near Grandview, WA & 12509057 & 46.1977 & -119.9186 \\
Joint drain 14.6 at Chervon Station at Zillah, WA & 1250532310 & 46.4041 & -120.2768 \\
Joint drain 28 near Granger, WA & 12505448 & 46.3397 & -120.1414 \\
Joint drain 32 at Outlook Road Near Sunnyside, WA & 12504509 & 46.3316 & -120.1044 \\
Joint drain 40.2 near Tear Road Near Sunnyside, WA & 12508825 & 46.2875 & -119.9975 \\
Joint drain at Yakima Valley Highway at Granger, WA & 12505445 & 46.3474 & -120.1775 \\
Joint drain from Rougk Lane near Sunnyside, WA & 12508835 & 46.3333 & -120.0332 \\
Joint drain near S. 1st Street at Sunnyside, WA & 12508785 & 46.3251 & -120.0203 \\
Roza Canal Wasteway Number 3 near Sawyer, WA & 12505150 & 46.4593 & -120.3698 \\
Sulphur Creek Wasteway at Sheller Road at Sunnyside WA & 12508788 & 46.3317 & -119.9800 \\
Sulphur Creek Wasteway near Sunnyside, WA & 12508850 & 46.2507 & -120.0198 \\
Washout Drain at Sunnyside, WA & 12508810 & 46.3096 & -119.9912 \\
\hline
\end{tabular}

Table 3. Numbers of environmental and quality-assurance samples collected from wells and drains in the lower Yakima River Basin, Groundwater Management Area, Yakima County, Washington.

[Number in parentheses is the percentage of samples from within the group]

\begin{tabular}{lrr|rr|rr}
\hline \multirow{2}{*}{ Sample type } & \multicolumn{4}{c}{ Number and (percentage) of samples } \\
\cline { 2 - 7 } & \multicolumn{2}{c|}{ Well } & \multicolumn{2}{|c}{ Drain } & \multicolumn{2}{c}{ Total } \\
\hline Environmental & 892 & $(92)$ & 164 & $(86)$ & 1,056 & $(91)$ \\
Field blanks & 22 & $(2)$ & 5 & $(3)$ & 27 & $(2)$ \\
Equipment blanks & 7 & $(1)$ & 6 & $(3)$ & 13 & $(1)$ \\
Replicates & 47 & $(5)$ & 15 & $(8)$ & 62 & $(5)$ \\
Total & 968 & $(100)$ & 190 & $(100)$ & 1,158 & $(100)$ \\
\hline
\end{tabular}

\section{Laboratory Methods}

Samples for analysis of nitrate plus nitrite were analyzed colorimetrically by enzymatic reduction as described by Patton and Kryskalla (2011). The results for the nitrate plus nitrite analyses are referred to simply as "nitrate" in this report because nitrite was below the detection level at all sites during the first round of sampling collection. 


\section{Results}

\section{Quality Control Results}

Quality-assurance sampling included collecting replicate and blank samples for the analysis of nitrate. The relative percent difference between these replicate samples ranged from 0 to 10 percent. Nitrate was not detected in any blank samples with the exception of one sample with a concentration of $0.043 \mathrm{mg} / \mathrm{L}$, just above the reporting limit of $0.04 \mathrm{mg} / \mathrm{L}$. No changes were made to the dataset based on these qualitycontrol data.

During the second round of sampling in May and June, there was a mix-up of samples either by the laboratory, or field personnel. It was undetermined which was the issue, resulting in the rejection of 16 results from that sampling round. It was evident that sample results were incorrect (actually reversed) based on the trending result in the groundwater well. In these cases, where it was obvious the results were incorrect and the nitrate value was greater than four standard deviations of the sample mean, the result was rejected.

\section{Results of Water-Quality Analysis}

Results of nitrate concentrations for individual samples collected in the GWMA from April to December 2017 are listed in table 4 for groundwater wells and table 5 for surface-water drain sites. All analytical results, including field measurements are available from the USGS NWIS web site https:/waterdata.usgs.gov/wa/nwis/.

Nitrate concentrations in groundwater samples ranged from less than 0.04 to $45.2 \mathrm{mg} / \mathrm{L}$ and less than 0.04 to 25.2 $\mathrm{mg} / \mathrm{L}$ in surface water from drain sites. The average nitrate concentration for groundwater samples was $6.1 \mathrm{mg} / \mathrm{L}$ as nitrogen. Concentrations of nitrate averaged $5.5 \mathrm{mg} / \mathrm{L}$ in drain site samples. The $10 \mathrm{mg} / \mathrm{L}$ maximum contaminant level (MCL) established by the EPA for drinking water was exceeded by 20 percent of samples from wells exceeded in 13 percent of samples from drain sites (table 6). At least one nitrate concentration above the MCL was detected in 26 percent of wells and 33 percent of drain sites sampled. Nitrate was not detected in 13 percent of all samples (below the method detection level of $0.04 \mathrm{mg} / \mathrm{L}), 15$ percent of well samples, and 5 percent of drain site samples.

Table 4. Nitrate concentrations in groundwater samples collected from drinking water wells in the lower Yakima River Basin, Groundwater Management Area, Yakima County, Washington, April-December, 2017.

[All values in milligrams per liter. Abbreviations: $<$, less than; -, no data; -*, no data (sample tap winterized; - **, no data (could not access property_ no permission); R, result value reviewed and rejected]

\begin{tabular}{|c|c|c|c|c|c|c|}
\hline Well No. & April/May & May/June & July & September & October & December \\
\hline 08N/22E-11L02 & 16.1 & 14.3 & 14.2 & 13.6 & 15.7 & 15.4 \\
\hline 08N/23E-01F02 & 0.247 & 0.292 & 0.53 & 0.594 & 0.936 & 0.522 \\
\hline 08N/23E-01H02 & 0.443 & 1.15 & 1.41 & 1.94 & 1.83 & 4.1 \\
\hline 08N/23E-01J01 & $<0.040$ & $<0.040$ & $<0.040$ & $<0.040$ & $<0.040$ & $<0.040$ \\
\hline 08N/23E-05G01 & 11.2 & - & - & - & - & - \\
\hline 08N/23E-06H02 & 10.3 & 7.66 & 7.36 & 7.49 & 8.37 & 7.97 \\
\hline 08N/23E-08E01 & 14.3 & 10.8 & 16.2 & 17.7 & 17.7 & 19.7 \\
\hline 08N/23E-10G01 & 11 & 11.4 & 11.2 & 11.3 & 11.3 & -* \\
\hline 08N/23E-11R01 & 0.681 & 1.37 & 2.27 & 1.75 & 1.16 & 0.715 \\
\hline 08N/23E-13B01 & 2.6 & 3.54 & 4.12 & 4.93 & 5.19 & 3.67 \\
\hline 09N/22E-01G02 & 11.5 & 10.9 & 10.8 & 10.5 & 11.1 & 11.6 \\
\hline 09N/22E-02D01 & 2.28 & 2.18 & 2 & 2.04 & 2.29 & 2.36 \\
\hline 09N/22E-03R01 & 4.77 & 4.56 & 4.54 & 4.58 & 6.94 & 9.13 \\
\hline 09N/22E-04B01 & 2.37 & 1.05 & 2.55 & 1.02 & 0.79 & 0.739 \\
\hline 09N/22E-05Q01 & $<0.040$ & $<0.040$ & $<0.040$ & $<0.040$ & $<0.040$ & $<0.040$ \\
\hline 09N/22E-09J02 & $<0.040$ & $<0.040$ & $<0.040$ & $<0.040$ & $<0.040$ & $<0.040$ \\
\hline 09N/22E-10A01 & $<0.040$ & $<0.040$ & $<0.040$ & $<0.040$ & $<0.040$ & $<0.040$ \\
\hline 09N/22E-10N03 & - & 0.47 & 0.322 & 0.075 & $<0.040$ & $<0.040$ \\
\hline 09N/22E-10N04 & - & - & $<0.040$ & $<0.040$ & $<0.040$ & $<0.040$ \\
\hline 09N/22E-11D01 & $<0.040$ & $<0.040$ & $<0.040$ & $<0.040$ & $<0.040$ & $<0.040$ \\
\hline 09N/22E-11M01 & 8.02 & 7.13 & 7.11 & 8.89 & 8.87 & 8.93 \\
\hline 09N/22E-12R02 & 14.5 & 16.4 & 17.9 & 18 & 17.9 & 17.9 \\
\hline 09N/22E-14B01 & $<0.040$ & 0.057 & $<0.040$ & $<0.040$ & $<0.040$ & $<0.040$ \\
\hline 09N/22E-22K01 & $<0.040$ & $<0.040$ & $<0.040$ & $<0.040$ & $<0.040$ & $<0.040$ \\
\hline 09N/22E-23J01 & $<0.040$ & $<0.040$ & $<0.040$ & $<0.040$ & $<0.040$ & $<0.040$ \\
\hline 09N/23E-01D01 & 2.27 & 2.18 & 2.16 & 2.23 & 2.34 & 2.57 \\
\hline 09N/23E-04R01 & 4.42 & 4.44 & 4.4 & 4.62 & 4.51 & 4.49 \\
\hline
\end{tabular}


Table 4. Nitrate concentrations in groundwater samples collected from drinking water wells in the lower Yakima River Basin, Groundwater Management Area, Yakima County, Washington, April-December, 2017.Continued

\begin{tabular}{|c|c|c|c|c|c|c|}
\hline Well No. & April/May & May/June & July & September & October & December \\
\hline 09N/23E-04R02 & 4.17 & 4.12 & 4.12 & 4.25 & 4.2 & 4.2 \\
\hline 09N/23E-05N01 & 5.39 & 5.29 & 5.29 & 5.45 & 5.31 & 5.42 \\
\hline 09N/23E-06B01 & 17.3 & 17.4 & 17 & 17.3 & 16.9 & -* \\
\hline 09N/23E-07M02 & $<0.040$ & $<0.040$ & $<0.040$ & $<0.040$ & $<0.040$ & $<0.040$ \\
\hline 09N/23E-08E02 & 7.19 & 7.42 & 7.62 & 7.92 & 7.87 & 7.8 \\
\hline 09N/23E-09H01 & $<0.040$ & $<0.040$ & $<0.040$ & $<0.040$ & $<0.040$ & $<0.040$ \\
\hline 09N/23E-11K01 & 6.23 & 6.43 & 6.29 & 6.58 & 6.37 & 6.4 \\
\hline $09 \mathrm{~N} / 23 \mathrm{E}-13 \mathrm{C} 01$ & 10.3 & 10.7 & 10.4 & 10.7 & 10.7 & 11.1 \\
\hline 09N/23E-14G01 & 14.2 & 15.1 & 17.3 & 24.1 & 32.9 & -** \\
\hline 09N/23E-15D03 & 12.8 & 13 & 12.8 & 12.7 & 12.8 & 12.8 \\
\hline $09 \mathrm{~N} / 23 \mathrm{E}-15 \mathrm{H} 03$ & 5.87 & 5.27 & 4.54 & 5.6 & 5.57 & 5.73 \\
\hline 09N/23E-16C01D1 & 2.62 & 2.66 & 2.58 & 2.57 & 2.62 & -* \\
\hline 09N/23E-17L01 & $\mathrm{R}$ & 19.2 & 19.3 & 20.7 & 19.8 & $-^{*}$ \\
\hline 09N/23E-18C01 & 1.89 & 1.72 & 1.71 & 1.69 & 1.88 & 1.85 \\
\hline 09N/23E-19D03 & 8.07 & 8.29 & 8.26 & 8.6 & - & - \\
\hline 09N/23E-19Q01 & 2.42 & 2.19 & 2.13 & 2.36 & 2.38 & 2.44 \\
\hline 09N/23E-20A01 & 5.02 & 4.73 & 3.92 & 3.57 & 3.61 & 3.62 \\
\hline 09N/23E-21P01 & 9.3 & 9.5 & 9.47 & 10.1 & 10.3 & 10.4 \\
\hline 09N/23E-24L01 & 8.2 & 5.26 & 5.13 & 7.52 & 7.69 & 8.25 \\
\hline 09N/23E-25J01 & 12 & 12.3 & 13 & 11.1 & 10.1 & 12.8 \\
\hline 09N/23E-26B01 & 2.51 & 3.92 & 4.1 & 4.64 & 2.67 & 2.58 \\
\hline 09N/23E-27B02 & 6.13 & 5.94 & 5.6 & 5.41 & 5.34 & 5.65 \\
\hline 09N/23E-28G01 & 3.58 & 3.68 & 3.75 & 3.93 & 3.9 & 3.84 \\
\hline 09N/23E-29B02 & 4.56 & 4.66 & 4.57 & 4.78 & 4.73 & 4.89 \\
\hline 09N/23E-31K01 & 8.41 & 7.98 & 8.63 & 10.7 & 10.7 & 10.6 \\
\hline 09N/23E-34M01 & $<0.040$ & $<0.040$ & $<0.040$ & $<0.040$ & $<0.040$ & $<0.040$ \\
\hline 09N/23E-35K01 & $<0.040$ & $<0.040$ & $<0.040$ & $<0.040$ & $<0.040$ & $<0.040$ \\
\hline 09N/23E-36J01 & 9.84 & 5.54 & 5.36 & 7.19 & 6.25 & -* $^{*}$ \\
\hline $10 \mathrm{~N} / 21 \mathrm{E}-01 \mathrm{G} 01$ & 43.1 & $\mathrm{R}$ & 40.8 & 41.7 & 39.4 & 42.5 \\
\hline 10N/21E-02N01 & 11.1 & $\mathrm{R}$ & 7.04 & 7.6 & 7.68 & 10.3 \\
\hline $10 \mathrm{~N} / 21 \mathrm{E}-03 \mathrm{D} 02$ & 11.6 & 12.4 & 12.4 & 12.2 & 11.5 & -* \\
\hline 10N/21E-04P02 & 3.72 & $\mathrm{R}$ & 3.58 & 3.79 & 3.75 & 3.88 \\
\hline $10 \mathrm{~N} / 21 \mathrm{E}-05 \mathrm{~A} 01$ & 4.63 & 4.05 & 4.36 & 5 & 4.67 & 5.28 \\
\hline 10N/21E-09F01 & 2.56 & 1.7 & 2.18 & 2.73 & 2.58 & 2.88 \\
\hline 10N/21E-11M01 & 11.1 & 8.27 & 8.63 & 8.76 & 8.26 & 11.3 \\
\hline 10N/21E-12R01 & 15 & 15.7 & 15.5 & 16.2 & 15.6 & 15.9 \\
\hline $10 \mathrm{~N} / 21 \mathrm{E}-13 \mathrm{~N} 01$ & $<0.040$ & 0.12 & 0.211 & $<0.040$ & $<0.040$ & $<0.040$ \\
\hline $10 \mathrm{~N} / 21 \mathrm{E}-15 \mathrm{E} 01$ & 2.87 & $\mathrm{R}$ & 3.05 & 3.19 & 3.01 & 3.14 \\
\hline $10 \mathrm{~N} / 21 \mathrm{E}-16 \mathrm{~B} 01$ & 14.8 & $\mathrm{R}$ & 15.5 & 15.5 & 13.2 & 12.7 \\
\hline $10 \mathrm{~N} / 21 \mathrm{E}-16 \mathrm{G} 02$ & 10.8 & 10.5 & 9.82 & 9.87 & 9.98 & 10.7 \\
\hline 10N/21E-23A01 & $<0.040$ & $<0.040$ & $<0.040$ & $<0.040$ & $<0.040$ & $<0.040$ \\
\hline 10N/21E-24J01 & $<0.040$ & $\mathrm{R}$ & $<0.040$ & $<0.040$ & $<0.040$ & $<0.040$ \\
\hline $10 \mathrm{~N} / 22 \mathrm{E}-01 \mathrm{~F} 01$ & 4.77 & 3.94 & 4.02 & 4.24 & 4.2 & -* \\
\hline $10 \mathrm{~N} / 22 \mathrm{E}-03 \mathrm{~B} 02$ & 45.2 & 43.2 & 44 & 44.5 & 43.1 & 44.7 \\
\hline 10N/22E-04J01 & 3.93 & $\mathrm{R}$ & 3.83 & 3.85 & 3.69 & 3.92 \\
\hline $10 \mathrm{~N} / 22 \mathrm{E}-05 \mathrm{P} 01$ & 8.14 & $\mathrm{R}$ & 7.66 & 7.98 & 8.04 & 8.56 \\
\hline $10 \mathrm{~N} / 22 \mathrm{E}-06 \mathrm{~A} 01$ & 5.05 & 6.38 & 6 & 6.56 & 4.85 & 4.94 \\
\hline 10N/22E-07N01 & 15.8 & 15.8 & 16.9 & 16.5 & 15.5 & 15.8 \\
\hline $10 \mathrm{~N} / 22 \mathrm{E}-08 \mathrm{~F} 02$ & 9.91 & 9.26 & 9.96 & 9.98 & 9.48 & 10.3 \\
\hline 10N/22E-08H01 & 6.05 & 5.79 & 6.42 & 6.77 & 6.82 & 6.83 \\
\hline $10 \mathrm{~N} / 22 \mathrm{E}-08 \mathrm{~K} 04$ & 9.66 & 8.27 & 9.31 & 9.36 & 8.95 & 9.09 \\
\hline 10N/22E-08L01 & 8.42 & 8.35 & 8.44 & 8.76 & 8.37 & 8.92 \\
\hline $10 \mathrm{~N} / 22 \mathrm{E}-11 \mathrm{~J} 02$ & 10.8 & 10.5 & 11 & 8.31 & 8.7 & 10.4 \\
\hline $10 \mathrm{~N} / 22 \mathrm{E}-13 \mathrm{E} 02$ & 7.11 & $\mathrm{R}$ & 6.88 & 6.73 & 6.73 & 7.16 \\
\hline
\end{tabular}


Table 4. Nitrate concentrations in groundwater samples collected from drinking water wells in the lower Yakima River Basin, Groundwater Management Area, Yakima County, Washington, April-December, 2017.Continued

\begin{tabular}{|c|c|c|c|c|c|c|}
\hline Well No. & April/May & May/June & July & September & October & December \\
\hline $10 \mathrm{~N} / 22 \mathrm{E}-14 \mathrm{~K} 01$ & 3.56 & 3.51 & 4.61 & 4.88 & 4.4 & 3.83 \\
\hline $10 \mathrm{~N} / 22 \mathrm{E}-17 \mathrm{C} 02$ & 12.1 & 9.76 & 8.41 & 10.2 & 18.9 & 18.1 \\
\hline $10 \mathrm{~N} / 22 \mathrm{E}-18 \mathrm{G} 03$ & 9.41 & 9.3 & 9.16 & 9.04 & 9.03 & 9.01 \\
\hline $10 \mathrm{~N} / 22 \mathrm{E}-19 \mathrm{~L} 01$ & $<0.040$ & $<0.040$ & $<0.040$ & $<0.040$ & $<0.040$ & $<0.040$ \\
\hline $10 \mathrm{~N} / 22 \mathrm{E}-20 \mathrm{~N} 02$ & $<0.040$ & $<0.040$ & $<0.040$ & $<0.040$ & - & - \\
\hline $10 \mathrm{~N} / 22 \mathrm{E}-21 \mathrm{R} 02$ & $<0.040$ & $<0.040$ & $<0.040$ & $<0.040$ & $<0.040$ & $<0.040$ \\
\hline $10 \mathrm{~N} / 22 \mathrm{E}-22 \mathrm{P} 01$ & 10.4 & 10.8 & 10.7 & 10.9 & 11 & 11 \\
\hline $10 \mathrm{~N} / 22 \mathrm{E}-23 \mathrm{H} 02$ & 4.93 & 4.98 & 4.96 & 5.08 & 5.04 & 5 \\
\hline $10 \mathrm{~N} / 22 \mathrm{E}-24 \mathrm{M} 01$ & 3.29 & 3.73 & 3.76 & 3.73 & 3.6 & 3.78 \\
\hline $10 \mathrm{~N} / 22 \mathrm{E}-26 \mathrm{C} 01$ & 0.402 & 0.283 & 0.342 & 0.352 & 0.395 & 0.465 \\
\hline $10 \mathrm{~N} / 22 \mathrm{E}-27 \mathrm{M} 01$ & 10.5 & $\mathrm{R}$ & 10.8 & 10.4 & 10.6 & 10.8 \\
\hline $10 \mathrm{~N} / 22 \mathrm{E}-27 \mathrm{~N} 01$ & 5.91 & 5.61 & 5.83 & 5.81 & 5.92 & 5.79 \\
\hline $10 \mathrm{~N} / 22 \mathrm{E}-29 \mathrm{D} 01$ & 10.5 & 10.3 & 10.1 & 10.2 & 10.2 & 11.3 \\
\hline $10 \mathrm{~N} / 22 \mathrm{E}-30 \mathrm{~B} 01$ & 5.87 & $\mathrm{R}$ & 5.08 & 5.37 & 5.73 & 5.89 \\
\hline $10 \mathrm{~N} / 22 \mathrm{E}-34 \mathrm{~B} 01$ & 14.9 & 17.2 & 16.9 & 15.5 & 12.7 & 9.67 \\
\hline $10 \mathrm{~N} / 22 \mathrm{E}-34 \mathrm{~B} 02$ & 8.55 & 8.56 & 8.46 & 8.52 & 8.62 & 8.79 \\
\hline $10 \mathrm{~N} / 22 \mathrm{E}-35 \mathrm{~F} 03$ & 2.16 & 2.11 & 2.13 & 2.04 & 2.1 & 2.09 \\
\hline $10 \mathrm{~N} / 22 \mathrm{E}-36 \mathrm{~K} 01$ & 17 & 18.2 & 16.1 & 11.4 & 9.87 & 10.7 \\
\hline $10 \mathrm{~N} / 23 \mathrm{E}-18 \mathrm{D} 01$ & 3.52 & 3.66 & 3.88 & 3.83 & 3.84 & 3.81 \\
\hline $10 \mathrm{~N} / 23 \mathrm{E}-20 \mathrm{G} 01$ & 8.78 & 8.77 & 8.28 & 7.71 & 7.84 & 8.09 \\
\hline 10N/23E-22L01 & 6.36 & 5.75 & 5.54 & 5.33 & 5.41 & 5.59 \\
\hline 10N/23E-23R01 & 4.46 & 4.26 & 12.9 & 21.7 & 19.5 & 11.3 \\
\hline 10N/23E-25J07 & 2.76 & 2.47 & 2.84 & 2.65 & 3.05 & 2.51 \\
\hline $10 \mathrm{~N} / 23 \mathrm{E}-27 \mathrm{~N} 01$ & 2.17 & 3.28 & 3.43 & 2.62 & 1.99 & 2.07 \\
\hline $10 \mathrm{~N} / 23 \mathrm{E}-28 \mathrm{~F} 01$ & 4.15 & 3.72 & 3.87 & 3.88 & 4.51 & 4.56 \\
\hline 10N/23E-29A01 & 9.2 & 9.56 & 9.77 & 9.45 & 9.77 & 9.86 \\
\hline $10 \mathrm{~N} / 23 \mathrm{E}-30 \mathrm{~A} 01$ & 19.4 & 20.6 & 21.6 & 21.7 & 21 & 22.3 \\
\hline 10N/23E-31E02 & 12.5 & 12.8 & 12.8 & 16 & 12.7 & 13.4 \\
\hline $10 \mathrm{~N} / 23 \mathrm{E}-32 \mathrm{~K} 02$ & 4.05 & $\mathrm{R}$ & 4.76 & 5.73 & 4.17 & 4.14 \\
\hline 10N/23E-33D01 & 9.21 & 9.82 & 9.67 & 10.2 & 9.49 & 9.82 \\
\hline $10 \mathrm{~N} / 23 \mathrm{E}-34 \mathrm{~A} 01$ & 10.3 & 10.2 & 11.3 & 11.6 & 12.4 & 14.2 \\
\hline 10N/23E-35M01 & 9.28 & 11.4 & 11.7 & 9.75 & 10.1 & 10.2 \\
\hline 11N/20E-04Q03D1 & 0.748 & 0.773 & 0.749 & 0.767 & 0.772 & -* $^{*}$ \\
\hline 11N/20E-06D01 & 7.24 & 7.31 & 7.56 & 8.1 & 7.63 & 7.6 \\
\hline $11 \mathrm{~N} / 20 \mathrm{E}-07 \mathrm{C} 01$ & 3.29 & 3.13 & 3.27 & 3.17 & 3.57 & 3.44 \\
\hline $11 \mathrm{~N} / 20 \mathrm{E}-07 \mathrm{H} 03$ & 2.46 & 2.43 & 2.39 & 2.34 & 2.4 & 2.55 \\
\hline $11 \mathrm{~N} / 20 \mathrm{E}-08 \mathrm{~F} 01$ & 4.72 & 4.42 & 4.28 & 3.91 & 4.17 & -* \\
\hline 11N/20E-09D02 & 2.05 & 2.25 & 2.17 & 2.07 & 2.18 & 2.15 \\
\hline 11N/20E-09L02 & 3.4 & 3.35 & 3.71 & 3.5 & 3.88 & 3.32 \\
\hline $11 \mathrm{~N} / 20 \mathrm{E}-10 \mathrm{C} 02$ & 1.76 & 1.21 & 1.1 & 1.03 & 1.26 & 1.53 \\
\hline 11N/20E-10P01 & 2.19 & 2.23 & 2.13 & 1.99 & 2.18 & 2.13 \\
\hline $11 \mathrm{~N} / 20 \mathrm{E}-11 \mathrm{R} 01$ & 2.46 & 2.66 & 2.57 & 2.47 & 2.75 & 2.48 \\
\hline $11 \mathrm{~N} / 20 \mathrm{E}-12 \mathrm{P} 02$ & 3.23 & 3.2 & 3.17 & 3.05 & 3.21 & 3.25 \\
\hline 11N/20E-13J01 & 2 & 1.96 & 1.86 & 1.76 & 1.77 & 1.92 \\
\hline $11 \mathrm{~N} / 20 \mathrm{E}-14 \mathrm{M} 03$ & 6.25 & 6.31 & 6.27 & 6.15 & 5.43 & 5.48 \\
\hline $11 \mathrm{~N} / 20 \mathrm{E}-15 \mathrm{~B} 02$ & 1.38 & 1.37 & 1.45 & 1.47 & 1.58 & 1.57 \\
\hline $11 \mathrm{~N} / 20 \mathrm{E}-21 \mathrm{~B} 02$ & 2.44 & 2.43 & 2.79 & 2.06 & 2.48 & 2.56 \\
\hline $11 \mathrm{~N} / 20 \mathrm{E}-22 \mathrm{Q} 01$ & 1.35 & 1.33 & 1.34 & 1.29 & 1.38 & $-*$ \\
\hline $11 \mathrm{~N} / 20 \mathrm{E}-23 \mathrm{Q} 02$ & 1.64 & 1.69 & 1.56 & 1.46 & 1.53 & 1.66 \\
\hline $11 \mathrm{~N} / 20 \mathrm{E}-24 \mathrm{E} 02$ & 7.4 & 7.29 & 7.38 & 7.5 & 7.38 & 7.41 \\
\hline $11 \mathrm{~N} / 20 \mathrm{E}-24 \mathrm{~J} 03$ & 5.08 & 4.95 & 4.92 & 4.63 & 4.9 & 4.99 \\
\hline $11 \mathrm{~N} / 20 \mathrm{E}-24 \mathrm{~N} 01$ & 5.44 & 5.31 & 5.34 & 4.99 & 5.4 & 5.56 \\
\hline $11 \mathrm{~N} / 20 \mathrm{E}-24 \mathrm{P} 03$ & 5.01 & 4.77 & 5.27 & 4.96 & 5.6 & 5.53 \\
\hline $11 \mathrm{~N} / 20 \mathrm{E}-24 \mathrm{R} 01$ & - & 2.86 & 2.87 & 2.61 & 2.72 & 2.75 \\
\hline
\end{tabular}


Table 4. Nitrate concentrations in groundwater samples collected from drinking water wells in the lower Yakima River Basin, Groundwater Management Area, Yakima County, Washington, April-December, 2017.Continued

\begin{tabular}{lcccccc}
\hline \multicolumn{1}{c}{ Well No. } & April/May & May/June & July & September & October & December \\
\hline $11 \mathrm{~N} / 20 \mathrm{E}-25 \mathrm{~L} 01$ & 3.91 & 3.95 & 3.96 & 3.56 & 4.04 & 4.07 \\
$11 \mathrm{~N} / 20 \mathrm{E}-26 \mathrm{c} 01$ & - & 2.32 & 2.28 & 2.13 & 2.23 & $-*$ \\
$11 \mathrm{~N} / 21 \mathrm{E}-06 \mathrm{R} 01 \mathrm{D} 1$ & $<0.040$ & $<0.040$ & $<0.040$ & $<0.040$ & $<0.040$ & $<0.040$ \\
$11 \mathrm{~N} / 21 \mathrm{E}-18 \mathrm{G} 01$ & 2.48 & $\mathrm{R}$ & 2.35 & 2.27 & 2.28 & 2.23 \\
$11 \mathrm{~N} / 21 \mathrm{E}-19 \mathrm{~N} 01$ & 3.11 & 3.08 & 3.08 & 3.01 & 3.14 & 3.11 \\
$11 \mathrm{~N} / 21 \mathrm{E}-20 \mathrm{~N} 01$ & 1.98 & 2.13 & 2.12 & 2.2 & 2.31 & 2.32 \\
$11 \mathrm{~N} / 21 \mathrm{E}-21 \mathrm{~N} 01$ & 2.09 & 2.03 & 1.99 & 1.84 & 1.99 & 1.96 \\
$11 \mathrm{~N} / 21 \mathrm{E}-21 \mathrm{~N} 02$ & 1.33 & 1.33 & 1.29 & 1.21 & 1.25 & 1.35 \\
$11 \mathrm{~N} / 21 \mathrm{E}-27 \mathrm{~A} 01$ & 1.22 & 5.46 & 0.078 & 4.98 & 5.47 & 5.74 \\
$11 \mathrm{~N} / 21 \mathrm{E}-28 \mathrm{H} 01$ & 2.43 & 2.25 & 2.41 & 2.41 & 2.32 & 2.37 \\
$11 \mathrm{~N} / 21 \mathrm{E}-29 \mathrm{M} 05$ & 8.55 & 8.46 & 8.41 & 8.61 & 8.15 & 8.26 \\
$11 \mathrm{~N} / 21 \mathrm{E}-30 \mathrm{~F} 03$ & 17.6 & 17.6 & 17.3 & 17.3 & 17.7 & 17.5 \\
$11 \mathrm{~N} / 21 \mathrm{E}-31 \mathrm{D} 01$ & 3.1 & 2.93 & 2.9 & 2.82 & 2.9 & 2.96 \\
$11 \mathrm{~N} / 21 \mathrm{E}-32 \mathrm{~N} 01$ & 7.99 & $\mathrm{R}$ & 8.46 & 8.65 & 7.97 & 8.7 \\
$11 \mathrm{~N} / 21 \mathrm{E}-33 \mathrm{C} 02$ & 9.72 & $\mathrm{R}$ & 9.79 & 10 & 9.8 & 10.3 \\
$11 \mathrm{~N} / 21 \mathrm{E}-33 \mathrm{M} 01$ & 6.56 & $\mathrm{R}$ & 6.58 & 6.99 & 6.44 & 7.05 \\
$12 \mathrm{~N} / 19 \mathrm{E}-27 \mathrm{Q} 01$ & $<0.040$ & $<0.040$ & $<0.040$ & $<0.040$ & $<0.040$ & $<0.040$ \\
$12 \mathrm{~N} / 19 \mathrm{E}-35 \mathrm{E} 01$ & 1.75 & 3.12 & 1.19 & 1.95 & 0.961 & 1.71 \\
$12 \mathrm{~N} / 19 \mathrm{E}-36 \mathrm{D} 01$ & $<0.040$ & $\mathrm{R}$ & $<0.040$ & $<0.040$ & $<0.040$ & $<0.040$ \\
$12 \mathrm{~N} / 20 \mathrm{E}-31 \mathrm{~B} 02$ & $<0.040$ & $<0.040$ & $<0.040$ & $<0.040$ & $<0.040$ & $<0.040$ \\
$12 \mathrm{~N} / 20 \mathrm{E}-33 \mathrm{2} 02$ & - & 0.856 & 0.762 & 0.772 & 0.733 & 0.678 \\
\hline
\end{tabular}

Table 5. Nitrate concentrations in surface-water samples collected from drain sites in the lower Yakima River Basin, Groundwater Management Area, Yakima County, Washington, April-December 2017.

[All values in milligrams per liter. Abbreviations: <;,less than; D, site dry]

\begin{tabular}{|c|c|c|c|c|c|c|c|}
\hline Station name & April & May & June/August & August & September & October & December \\
\hline DID 18 drain at Sunnyside, WA & 9.32 & 6.41 & 5.59 & 5.57 & 6.34 & 9.42 & 9.87 \\
\hline DID 7 drain near Mabton, WA & 0.653 & 0.557 & 0.519 & $<0.040$ & 0.988 & 0.995 & 1.25 \\
\hline DR2 NEET Site Number 3 & 6.13 & 4.82 & 3 & 1.27 & 3.64 & 7.97 & 7.58 \\
\hline DRAIN 31 at West Charvet Road at Mabton, WA & 20.8 & 4.74 & 4.95 & 4.64 & 6.86 & 25.2 & 24.3 \\
\hline Granger Drain at Granger, WA & 5.28 & 3.91 & 2.47 & 3.08 & 2.87 & 7.19 & 7.66 \\
\hline Joint drain 27.5 at Vanbelle Road & 5.66 & 3.1 & 1.94 & 3.36 & 3.56 & 9.2 & 9.85 \\
\hline Joint drain 34.2 at Woodin Road & 10.7 & 5.88 & 5.01 & 4.64 & 4.03 & 15.9 & 16.5 \\
\hline Joint drain 43.9 at Mabton Sunnyside Road & 6.19 & 4.58 & 4.16 & 3.68 & 4.03 & 6.63 & 8.22 \\
\hline Joint drain 1 at Bus Road Near Grandview, WA & 4.35 & 7.13 & 6.11 & 6.27 & 13.6 & 18.6 & 12.6 \\
\hline Joint drain at Yakima Valley Highway at Granger, WA & 10.2 & 5.46 & 3.5 & 3.49 & 3.52 & 10.7 & 10.9 \\
\hline Joint drain from Rougk Lane near Sunnyside, WA & 4.14 & 4.09 & 2.53 & 1.35 & 3.08 & 7.78 & 9.06 \\
\hline Joint drain near S. 1st Street at Sunnyside, WA & 5.37 & 4.74 & 3.44 & 1.97 & 3.97 & 7.61 & 7.78 \\
\hline Roza Canal Wasteway Number 3 near Sawyer, WA & $<0.040$ & 0.072 & $<0.040$ & $<0.040$ & $<0.040$ & $\mathrm{D}$ & $\mathrm{D}$ \\
\hline Sulphur Creek Wasteway at Sheller Road at Sunnyside WA & $<0.040$ & 0.242 & 0.075 & $<0.040$ & 0.21 & $\mathrm{D}$ & $\mathrm{D}$ \\
\hline Sulphur Creek Wasteway near Sunnyside, WA & 3.25 & 4.65 & 3.91 & 2.43 & 2.4 & 7.84 & 9.2 \\
\hline Washout Drain at Sunnyside, WA & 9.24 & 8.3 & 5.7 & 5.82 & 6.31 & 10.8 & 11 \\
\hline
\end{tabular}


Table 6. Statistical summary of nitrate concentrations in groundwater and surface-water samples collected from domestic wells and drains in the lower Yakima River Basin, Groundwater Management Area, Yakima County, Washington.

[Minimum, Maximum, and Median: All values in milligrams per liter. Number of detections exceeding drinking water standard: The drinking water standard (maximum contaminant level) established by the U.S. Environmental Protection Agency for nitrate concentration is 10 milligrams per liter (U.S. Environmental Protection Agency, 2012). Remarks: -, no remark. Symbol: <, less than]

\begin{tabular}{|c|c|c|c|c|c|c|}
\hline $\begin{array}{c}\text { Well No. } \\
\text { or station name }\end{array}$ & Minimum & Maximum & Median & $\begin{array}{l}\text { Number of } \\
\text { samples }\end{array}$ & $\begin{array}{c}\text { Number of detections } \\
\text { exceeding drinking } \\
\text { water standard }\end{array}$ & Remarks \\
\hline \multicolumn{7}{|c|}{ Wells } \\
\hline 08N/22E-11L02 & 13.6 & 16.1 & 14.8 & 6 & 6 & - \\
\hline 08N/23E-01F02 & 0.247 & 0.936 & 0.526 & 6 & 0 & - \\
\hline 08N/23E-01H02 & 0.443 & 4.1 & 1.62 & 6 & 0 & - \\
\hline 08N/23E-01J01 & $<0.040$ & $<0.040$ & $<0.040$ & 6 & 0 & - \\
\hline 08N/23E-05G01 & 11.2 & 11.2 & 11.2 & 1 & 1 & Dropped from network in May \\
\hline 08N/23E-06H02 & 7.36 & 10.3 & 7.81 & 6 & 1 & - \\
\hline 08N/23E-08E01 & 10.8 & 19.7 & 16.9 & 6 & 6 & - \\
\hline 08N/23E-10G01 & 11 & 11.4 & 11.3 & 5 & 5 & - \\
\hline 08N/23E-11R01 & 0.681 & 2.27 & 1.26 & 6 & 0 & - \\
\hline 08N/23E-13B01 & 2.6 & 5.19 & 3.89 & 6 & 0 & - \\
\hline $09 \mathrm{~N} / 22 \mathrm{E}-01 \mathrm{G} 02$ & 10.5 & 11.6 & 11 & 6 & 6 & - \\
\hline 09N/22E-02D01 & 2 & 2.36 & 2.23 & 6 & 0 & - \\
\hline 09N/22E-03R01 & 4.54 & 9.13 & 4.67 & 6 & 0 & - \\
\hline 09N/22E-04B01 & 0.739 & 2.55 & 1.03 & 6 & 0 & - \\
\hline 09N/22E-05Q01 & $<0.040$ & $<0.040$ & $<0.040$ & 6 & 0 & - \\
\hline 09N/22E-09J02 & $<0.040$ & $<0.040$ & $<0.040$ & 6 & 0 & - \\
\hline $09 \mathrm{~N} / 22 \mathrm{E}-10 \mathrm{~A} 01$ & $<0.040$ & $<0.040$ & $<0.040$ & 6 & 0 & - \\
\hline 09N/22E-10N03 & $<0.040$ & 0.47 & 0.32 & 5 & 0 & Added to network in May \\
\hline 09N/22E-10N04 & $<0.040$ & $<0.040$ & $<0.040$ & 4 & 0 & Added to network in May \\
\hline 09N/22E-11D01 & $<0.040$ & $<0.040$ & $<0.040$ & 6 & 0 & - \\
\hline 09N/22E-11M01 & 7.11 & 8.93 & 8.44 & 6 & 0 & - \\
\hline 09N/22E-12R02 & 14.5 & 18 & 17.9 & 6 & 6 & - \\
\hline $09 \mathrm{~N} / 22 \mathrm{E}-14 \mathrm{~B} 01$ & $<0.040$ & 0.057 & $<0.040$ & 6 & 0 & - \\
\hline 09N/22E-22K01 & $<0.040$ & $<0.040$ & $<0.040$ & 6 & 0 & - \\
\hline 09N/22E-23J01 & $<0.040$ & $<0.040$ & $<0.040$ & 6 & 0 & - \\
\hline 09N/23E-01D01 & 2.16 & 2.57 & 2.25 & 6 & 0 & - \\
\hline 09N/23E-04R01 & 4.4 & 4.62 & 4.46 & 6 & 0 & - \\
\hline 09N/23E-04R02 & 4.12 & 4.25 & 4.18 & 6 & 0 & - \\
\hline 09N/23E-05N01 & 5.29 & 5.45 & 5.35 & 6 & 0 & - \\
\hline 09N/23E-06B01 & 16.9 & 17.4 & 17.3 & 5 & 5 & - \\
\hline 09N/23E-07M02 & $<0.040$ & $<0.040$ & $<0.040$ & 6 & 0 & - \\
\hline 09N/23E-08E02 & 7.19 & 7.92 & 7.71 & 6 & 0 & - \\
\hline 09N/23E-09H01 & $<0.040$ & $<0.040$ & $<0.040$ & 6 & 0 & - \\
\hline 09N/23E-11K01 & 6.23 & 6.58 & 6.38 & 6 & 0 & - \\
\hline 09N/23E-13C01 & 10.3 & 11.1 & 10.7 & 6 & 6 & - \\
\hline 09N/23E-14G01 & 14.2 & 32.9 & 17.3 & 5 & 5 & - \\
\hline 09N/23E-15D03 & 12.7 & 13 & 12.8 & 6 & 6 & - \\
\hline $09 \mathrm{~N} / 23 \mathrm{E}-15 \mathrm{H} 03$ & 4.54 & 5.87 & 5.58 & 6 & 0 & - \\
\hline 09N/23E-16C01D1 & 2.57 & 2.66 & 2.62 & 5 & 0 & - \\
\hline 09N/23E-17L01 & 19.2 & 20.7 & 19.5 & 4 & 4 & - \\
\hline $09 \mathrm{~N} / 23 \mathrm{E}-18 \mathrm{C} 01$ & 1.69 & 1.89 & 1.78 & 6 & 0 & - \\
\hline 09N/23E-19D03 & 8.07 & 8.6 & 8.27 & 4 & 0 & Dropped from network in October \\
\hline 09N/23E-19Q01 & 2.13 & 2.44 & 2.37 & 6 & 0 & - \\
\hline 09N/23E-20A01 & 3.57 & 5.02 & 3.77 & 6 & 0 & - \\
\hline 09N/23E-21P01 & 9.3 & 10.4 & 9.8 & 6 & 3 & - \\
\hline 09N/23E-24L01 & 5.13 & 8.25 & 7.6 & 6 & 0 & - \\
\hline 09N/23E-25J01 & 10.1 & 13 & 12.1 & 6 & 6 & - \\
\hline
\end{tabular}


Table 6. Statistical summary of nitrate concentrations in groundwater and surface-water samples collected from domestic wells and drains in the lower Yakima River Basin, Groundwater Management Area, Yakima County, Washington.—Continued

\begin{tabular}{|c|c|c|c|c|c|c|}
\hline $\begin{array}{c}\text { Well No. } \\
\text { or station name }\end{array}$ & Minimum & Maximum & Median & $\begin{array}{l}\text { Number of } \\
\text { samples }\end{array}$ & $\begin{array}{c}\text { Number of detections } \\
\text { exceeding drinking } \\
\text { water standard }\end{array}$ & Remarks \\
\hline \multicolumn{7}{|c|}{ Wells-Continued } \\
\hline $09 \mathrm{~N} / 23 \mathrm{E}-26 \mathrm{~B} 01$ & 2.51 & 4.64 & 3.29 & 6 & 0 & - \\
\hline 09N/23E-27B02 & 5.34 & 6.13 & 5.62 & 6 & 0 & - \\
\hline 09N/23E-28G01 & 3.58 & 3.93 & 3.79 & 6 & 0 & - \\
\hline 09N/23E-29B02 & 4.56 & 4.89 & 4.69 & 6 & 0 & - \\
\hline 09N/23E-31K01 & 7.98 & 10.7 & 9.61 & 6 & 3 & - \\
\hline 09N/23E-34M01 & $<0.040$ & $<0.040$ & $<0.040$ & 6 & 0 & - \\
\hline 09N/23E-35K01 & $<0.040$ & $<0.040$ & $<0.040$ & 6 & 0 & - \\
\hline 09N/23E-36J01 & 5.36 & 9.84 & 6.25 & 5 & 0 & - \\
\hline 10N/21E-01G01 & 39.4 & 43.1 & 41.7 & 5 & 5 & - \\
\hline $10 \mathrm{~N} / 21 \mathrm{E}-02 \mathrm{~N} 01$ & 7.04 & 15.4 & 8.99 & 6 & 3 & - \\
\hline $10 \mathrm{~N} / 21 \mathrm{E}-03 \mathrm{D} 02$ & 11.5 & 12.4 & 12.2 & 5 & 5 & - \\
\hline 10N/21E-04P02 & 3.58 & 3.88 & 3.75 & 5 & 0 & - \\
\hline $10 \mathrm{~N} / 21 \mathrm{E}-05 \mathrm{~A} 01$ & 4.05 & 5.28 & 4.65 & 6 & 0 & - \\
\hline 10N/21E-09F01 & 1.7 & 2.88 & 2.57 & 6 & 0 & - \\
\hline $10 \mathrm{~N} / 21 \mathrm{E}-11 \mathrm{M} 01$ & 8.26 & 11.3 & 8.69 & 6 & 2 & - \\
\hline 10N/21E-12R01 & 15 & 16.2 & 15.6 & 6 & 6 & - \\
\hline 10N/21E-13N01 & 0.12 & 0.211 & 0.165 & 6 & 0 & - \\
\hline 10N/21E-15E01 & 2.87 & 3.19 & 3.05 & 5 & 0 & - \\
\hline $10 \mathrm{~N} / 21 \mathrm{E}-16 \mathrm{~B} 01$ & 12.7 & 15.5 & 14.8 & 5 & 5 & - \\
\hline $10 \mathrm{~N} / 21 \mathrm{E}-16 \mathrm{G} 02$ & 9.82 & 10.8 & 10.2 & 6 & 3 & - \\
\hline $10 \mathrm{~N} / 21 \mathrm{E}-23 \mathrm{~A} 01$ & $<0.040$ & $<0.040$ & $<0.040$ & 6 & 0 & - \\
\hline 10N/21E-24J01 & $<0.040$ & $<0.040$ & $<0.040$ & 5 & 0 & - \\
\hline $10 \mathrm{~N} / 22 \mathrm{E}-01 \mathrm{~F} 01$ & 3.94 & 4.77 & 4.2 & 5 & 0 & - \\
\hline $10 \mathrm{~N} / 22 \mathrm{E}-03 \mathrm{~B} 02$ & 43.1 & 45.2 & 44.2 & 6 & 6 & - \\
\hline $10 \mathrm{~N} / 22 \mathrm{E}-04 \mathrm{~J} 01$ & 3.69 & 3.93 & 3.85 & 5 & 0 & - \\
\hline 10N/22E-05P01 & 7.66 & 8.56 & 8.04 & 5 & 0 & - \\
\hline $10 \mathrm{~N} / 22 \mathrm{E}-06 \mathrm{~A} 01$ & 4.85 & 6.56 & 5.52 & 6 & 0 & - \\
\hline 10N/22E-07N01 & 15.5 & 16.9 & 15.8 & 6 & 6 & - \\
\hline $10 \mathrm{~N} / 22 \mathrm{E}-08 \mathrm{~F} 02$ & 9.26 & 10.3 & 9.93 & 6 & 1 & - \\
\hline $10 \mathrm{~N} / 22 \mathrm{E}-08 \mathrm{H} 01$ & 5.79 & 6.83 & 6.59 & 6 & 0 & - \\
\hline $10 \mathrm{~N} / 22 \mathrm{E}-08 \mathrm{~K} 04$ & 8.27 & 9.66 & 9.2 & 6 & 0 & - \\
\hline 10N/22E-08L01 & 8.35 & 8.92 & 8.43 & 6 & 0 & - \\
\hline 10N/22E-11J02 & 8.31 & 11 & 10.4 & 6 & 4 & - \\
\hline $10 \mathrm{~N} / 22 \mathrm{E}-13 \mathrm{E} 02$ & 6.73 & 7.16 & 6.88 & 5 & 0 & - \\
\hline $10 \mathrm{~N} / 22 \mathrm{E}-14 \mathrm{~K} 01$ & 3.51 & 4.88 & 4.11 & 6 & 0 & - \\
\hline $10 \mathrm{~N} / 22 \mathrm{E}-17 \mathrm{C} 02$ & 8.41 & 18.9 & 11.1 & 6 & 4 & - \\
\hline $10 \mathrm{~N} / 22 \mathrm{E}-18 \mathrm{G} 03$ & 9.01 & 9.41 & 9.1 & 6 & 0 & - \\
\hline 10N/22E-19L01 & $<0.040$ & $<0.040$ & $<0.040$ & 6 & 0 & - \\
\hline $10 \mathrm{~N} / 22 \mathrm{E}-20 \mathrm{~N} 02$ & $<0.040$ & $<0.040$ & $<0.040$ & 4 & 0 & Dropped from network in October \\
\hline $10 \mathrm{~N} / 22 \mathrm{E}-21 \mathrm{R} 02$ & $<0.040$ & $<0.040$ & $<0.040$ & 6 & 0 & - \\
\hline $10 \mathrm{~N} / 22 \mathrm{E}-22 \mathrm{P} 01$ & 10.4 & 11 & 10.8 & 6 & 6 & - \\
\hline $10 \mathrm{~N} / 22 \mathrm{E}-23 \mathrm{H} 02$ & 4.93 & 5.08 & 4.99 & 6 & 0 & - \\
\hline $10 \mathrm{~N} / 22 \mathrm{E}-24 \mathrm{M} 01$ & 3.29 & 3.78 & 3.73 & 6 & 0 & - \\
\hline $10 \mathrm{~N} / 22 \mathrm{E}-26 \mathrm{C} 01$ & 0.283 & 0.465 & 0.373 & 6 & 0 & - \\
\hline 10N/22E-27M01 & 10.4 & 10.8 & 10.6 & 5 & 5 & - \\
\hline $10 \mathrm{~N} / 22 \mathrm{E}-27 \mathrm{~N} 01$ & 5.61 & 5.92 & 5.82 & 6 & 0 & - \\
\hline 10N/22E-29D01 & 10.1 & 11.3 & 10.2 & 6 & 6 & - \\
\hline $10 \mathrm{~N} / 22 \mathrm{E}-30 \mathrm{~B} 01$ & 5.08 & 5.89 & 5.73 & 5 & 0 & - \\
\hline $10 \mathrm{~N} / 22 \mathrm{E}-34 \mathrm{~B} 01$ & 9.67 & 17.2 & 15.2 & 6 & 5 & - \\
\hline $10 \mathrm{~N} / 22 \mathrm{E}-34 \mathrm{~B} 02$ & 8.46 & 8.79 & 8.55 & 6 & 0 & - \\
\hline
\end{tabular}


Table 6. Statistical summary of nitrate concentrations in groundwater and surface-water samples collected from domestic wells and drains in the lower Yakima River Basin, Groundwater Management Area, Yakima County, Washington.-Continued

\begin{tabular}{|c|c|c|c|c|c|c|}
\hline $\begin{array}{c}\text { Well No. } \\
\text { or station name }\end{array}$ & Minimum & Maximum & Median & $\begin{array}{l}\text { Number of } \\
\text { samples }\end{array}$ & $\begin{array}{l}\text { Number of detections } \\
\text { exceeding drinking } \\
\text { water standard }\end{array}$ & Remarks \\
\hline \multicolumn{7}{|c|}{ Wells-Continued } \\
\hline $10 \mathrm{~N} / 22 \mathrm{E}-35 \mathrm{~F} 03$ & 2.04 & 2.16 & 2.1 & 6 & 0 & - \\
\hline $10 \mathrm{~N} / 22 \mathrm{E}-36 \mathrm{~K} 01$ & 9.87 & 18.2 & 13.7 & 6 & 5 & - \\
\hline $10 \mathrm{~N} / 23 \mathrm{E}-18 \mathrm{D} 01$ & 3.52 & 3.88 & 3.82 & 6 & 0 & - \\
\hline $10 \mathrm{~N} / 23 \mathrm{E}-20 \mathrm{G} 01$ & 7.71 & 8.78 & 8.18 & 6 & 0 & - \\
\hline $10 \mathrm{~N} / 23 \mathrm{E}-22 \mathrm{~L} 01$ & 5.33 & 6.36 & 5.56 & 6 & 0 & - \\
\hline $10 \mathrm{~N} / 23 \mathrm{E}-23 \mathrm{R} 01$ & 4.26 & 21.7 & 12.1 & 6 & 4 & - \\
\hline $10 \mathrm{~N} / 23 \mathrm{E}-25 \mathrm{~J} 07$ & 2.47 & 3.05 & 2.7 & 6 & 0 & - \\
\hline $10 \mathrm{~N} / 23 \mathrm{E}-27 \mathrm{~N} 01$ & 1.99 & 3.43 & 2.39 & 6 & 0 & - \\
\hline $10 \mathrm{~N} / 23 \mathrm{E}-28 \mathrm{~F} 01$ & 3.72 & 4.56 & 4.01 & 6 & 0 & - \\
\hline $10 \mathrm{~N} / 23 \mathrm{E}-29 \mathrm{~A} 01$ & 9.2 & 9.86 & 9.66 & 6 & 0 & - \\
\hline $10 \mathrm{~N} / 23 \mathrm{E}-30 \mathrm{~A} 01$ & 19.4 & 22.3 & 21.3 & 6 & 6 & - \\
\hline $10 \mathrm{~N} / 23 \mathrm{E}-31 \mathrm{E} 02$ & 12.5 & 16 & 12.8 & 6 & 6 & - \\
\hline $10 \mathrm{~N} / 23 \mathrm{E}-32 \mathrm{~K} 02$ & 4.05 & 5.73 & 4.17 & 5 & 0 & - \\
\hline $10 \mathrm{~N} / 23 \mathrm{E}-33 \mathrm{D} 01$ & 9.21 & 10.2 & 9.74 & 6 & 1 & - \\
\hline $10 \mathrm{~N} / 23 \mathrm{E}-34 \mathrm{~A} 01$ & 10.2 & 14.2 & 11.4 & 6 & 6 & - \\
\hline $10 \mathrm{~N} / 23 \mathrm{E}-35 \mathrm{M} 01$ & 9.28 & 11.7 & 10.1 & 6 & 4 & - \\
\hline 11N/20E-04Q03D1 & 0.748 & 0.773 & 0.767 & 5 & 0 & - \\
\hline $11 \mathrm{~N} / 20 \mathrm{E}-06 \mathrm{D} 01$ & 7.24 & 8.1 & 7.58 & 6 & 0 & - \\
\hline $11 \mathrm{~N} / 20 \mathrm{E}-07 \mathrm{C} 01$ & 3.13 & 3.57 & 3.28 & 6 & 0 & - \\
\hline $11 \mathrm{~N} / 20 \mathrm{E}-07 \mathrm{H} 03$ & 2.34 & 2.55 & 2.41 & 6 & 0 & - \\
\hline $11 \mathrm{~N} / 20 \mathrm{E}-08 \mathrm{~F} 01$ & 3.91 & 4.72 & 4.28 & 5 & 0 & - \\
\hline $11 \mathrm{~N} / 20 \mathrm{E}-09 \mathrm{D} 02$ & 2.05 & 2.25 & 2.16 & 6 & 0 & - \\
\hline 11N/20E-09L02 & 3.32 & 3.88 & 3.45 & 6 & 0 & - \\
\hline $11 \mathrm{~N} / 20 \mathrm{E}-10 \mathrm{C} 02$ & 1.03 & 1.76 & 1.23 & 6 & 0 & - \\
\hline $11 \mathrm{~N} / 20 \mathrm{E}-10 \mathrm{P} 01$ & 1.99 & 2.23 & 2.15 & 6 & 0 & - \\
\hline $11 \mathrm{~N} / 20 \mathrm{E}-11 \mathrm{R} 01$ & 2.46 & 2.75 & 2.52 & 6 & 0 & - \\
\hline $11 \mathrm{~N} / 20 \mathrm{E}-12 \mathrm{P} 02$ & 3.05 & 3.25 & 3.2 & 6 & 0 & - \\
\hline 11N/20E-13J01 & 1.76 & 2 & 1.89 & 6 & 0 & - \\
\hline $11 \mathrm{~N} / 20 \mathrm{E}-14 \mathrm{M} 03$ & 5.43 & 6.31 & 6.2 & 6 & 0 & - \\
\hline $11 \mathrm{~N} / 20 \mathrm{E}-15 \mathrm{~B} 02$ & 1.37 & 1.58 & 1.46 & 6 & 0 & - \\
\hline $11 \mathrm{~N} / 20 \mathrm{E}-21 \mathrm{~B} 02$ & 2.06 & 2.79 & 2.46 & 6 & 0 & - \\
\hline $11 \mathrm{~N} / 20 \mathrm{E}-22 \mathrm{Q} 01$ & 1.29 & 1.38 & 1.34 & 5 & 0 & - \\
\hline $11 \mathrm{~N} / 20 \mathrm{E}-23 \mathrm{Q} 02$ & 1.46 & 1.69 & 1.6 & 6 & 0 & - \\
\hline $11 \mathrm{~N} / 20 \mathrm{E}-24 \mathrm{E} 02$ & 7.29 & 7.5 & 7.39 & 6 & 0 & - \\
\hline $11 \mathrm{~N} / 20 \mathrm{E}-24 \mathrm{~J} 03$ & 4.63 & 5.08 & 4.93 & 6 & 0 & - \\
\hline $11 \mathrm{~N} / 20 \mathrm{E}-24 \mathrm{~N} 01$ & 4.99 & 5.56 & 5.37 & 6 & 0 & - \\
\hline $11 \mathrm{~N} / 20 \mathrm{E}-24 \mathrm{P} 03$ & 4.77 & 5.6 & 5.14 & 6 & 0 & - \\
\hline $11 \mathrm{~N} / 20 \mathrm{E}-24 \mathrm{R} 01$ & 2.61 & 2.87 & 2.75 & 5 & 0 & Added to network in June \\
\hline $11 \mathrm{~N} / 20 \mathrm{E}-25 \mathrm{~L} 01$ & 3.56 & 4.07 & 3.95 & 6 & 0 & - \\
\hline $11 \mathrm{~N} / 20 \mathrm{E}-26 \mathrm{~F} 01$ & 2.13 & 2.32 & 2.25 & 4 & 0 & Added to network in July \\
\hline 11N/21E-06R01D1 & $<0.040$ & $<0.040$ & $<0.040$ & 6 & 0 & - \\
\hline 11N/21E-18G01 & 2.23 & 2.48 & 2.28 & 5 & 0 & - \\
\hline 11N/21E-19J01 & 3.01 & 3.14 & 3.09 & 6 & 0 & - \\
\hline $11 \mathrm{~N} / 21 \mathrm{E}-20 \mathrm{~N} 01$ & 1.98 & 2.32 & 2.16 & 6 & 0 & - \\
\hline $11 \mathrm{~N} / 21 \mathrm{E}-21 \mathrm{~N} 01$ & 1.84 & 2.09 & 1.99 & 6 & 0 & - \\
\hline $11 \mathrm{~N} / 21 \mathrm{E}-21 \mathrm{~N} 02$ & 1.21 & 1.35 & 1.31 & 6 & 0 & - \\
\hline $11 \mathrm{~N} / 21 \mathrm{E}-27 \mathrm{~A} 01$ & 0.078 & 5.74 & 5.22 & 6 & 0 & - \\
\hline 11N/21E-28H01 & 2.25 & 2.43 & 2.39 & 6 & 0 & - \\
\hline 11N/21E-29M05 & 8.15 & 8.61 & 8.43 & 6 & 0 & - \\
\hline $11 \mathrm{~N} / 21 \mathrm{E}-30 \mathrm{~F} 03$ & 17.3 & 17.7 & 17.55 & 6 & 6 & - \\
\hline $11 \mathrm{~N} / 21 \mathrm{E}-31 \mathrm{D} 01$ & 2.82 & 3.1 & 2.91 & 6 & 0 & - \\
\hline $11 \mathrm{~N} / 21 \mathrm{E}-32 \mathrm{~N} 01$ & 7.97 & 8.7 & 8.46 & 5 & 0 & - \\
\hline
\end{tabular}


Table 6. Statistical summary of nitrate concentrations in groundwater and surface-water samples collected from domestic wells and drains in the lower Yakima River Basin, Groundwater Management Area, Yakima County, Washington.-Continued

\begin{tabular}{|c|c|c|c|c|c|c|}
\hline $\begin{array}{l}\text { Well No. } \\
\text { or station name }\end{array}$ & Minimum & Maximum & Median & $\begin{array}{l}\text { Number of } \\
\text { samples }\end{array}$ & $\begin{array}{l}\text { Number of detections } \\
\text { exceeding drinking } \\
\text { water standard }\end{array}$ & Remarks \\
\hline \multicolumn{7}{|c|}{ Wells-Continued } \\
\hline $11 \mathrm{~N} / 21 \mathrm{E}-33 \mathrm{C} 02$ & 9.72 & 10.3 & 9.8 & 5 & 2 & - \\
\hline 11N/21E-33M01 & 6.44 & 7.05 & 6.58 & 5 & 0 & - \\
\hline 12N/19E-27Q01 & $<0.040$ & $<0.040$ & $<0.040$ & 6 & 0 & - \\
\hline 12N/19E-35E01 & 0.961 & 3.12 & 1.73 & 6 & 0 & - \\
\hline 12N/19E-36D01 & $<0.040$ & $<0.040$ & $<0.040$ & 5 & 0 & - \\
\hline $12 \mathrm{~N} / 20 \mathrm{E}-31 \mathrm{~B} 02$ & $<0.040$ & $<0.040$ & $<0.040$ & 6 & 0 & - \\
\hline \multirow[t]{2}{*}{$12 \mathrm{~N} / 20 \mathrm{E}-33 \mathrm{Q} 02$} & 0.678 & 0.856 & 0.762 & 5 & 0 & Added to network in May \\
\hline & & & Total & 892 & 181 & \\
\hline \multicolumn{7}{|c|}{ Surface-water drain sites } \\
\hline $\begin{array}{l}\text { Buena Drain at Westbound I- } 82 \\
\text { near Buena, WA }\end{array}$ & 0.177 & 2.61 & 0.83 & 7 & 0 & - \\
\hline Did 18 drain at Sunnyside, WA & 5.57 & 9.87 & 6.41 & 7 & 0 & - \\
\hline Did 3 drain near Sunnyside, WA & 3.07 & 12.3 & 8.22 & 7 & 2 & - \\
\hline Did 7 drain near Mabton, WA & $<0.040$ & 1.25 & 0.65 & 7 & 0 & - \\
\hline DR2 NEET Site Number 3 & 1.27 & 7.97 & 4.82 & 7 & 0 & - \\
\hline $\begin{array}{l}\text { Drain } 31 \text { at West Charvet Road at } \\
\text { Mabton, WA }\end{array}$ & 4.64 & 25.2 & 6.86 & 7 & 3 & - \\
\hline $\begin{array}{l}\text { Drain } 35 \text { off Charvet Road, near } \\
\text { Grandview, WA }\end{array}$ & 2.41 & 5.49 & 3.42 & 7 & 0 & - \\
\hline $\begin{array}{l}\text { Grandview Drain at Chase Road } \\
\text { near Grandview, WA }\end{array}$ & 3.94 & 6.98 & 6.24 & 7 & 0 & - \\
\hline Granger Drain at Granger, WA & 2.47 & 7.66 & 3.91 & 7 & 0 & - \\
\hline Joint drain 27.5 at Vanbelle Road & 1.94 & 9.85 & 3.56 & 7 & 0 & - \\
\hline Joint drain 34.2 at Woodin Road & 4.03 & 16.5 & 5.88 & 7 & 3 & - \\
\hline $\begin{array}{l}\text { Joint drain } 43.9 \text { at Mabton } \\
\text { Sunnyside Road }\end{array}$ & 3.68 & 8.22 & 4.58 & 7 & 0 & - \\
\hline $\begin{array}{l}\text { Joint drain } 1 \text { at Bus Road near } \\
\text { Grandview, WA }\end{array}$ & 4.35 & 18.6 & 7.13 & 7 & 3 & - \\
\hline $\begin{array}{l}\text { Joint drain } 14.6 \text { at Chervon } \\
\text { Station at Zillah, WA }\end{array}$ & $<0.040$ & 1.29 & 0.33 & 7 & 0 & - \\
\hline Joint drain 28 near Granger, WA & 3.37 & 9.98 & 5.96 & 7 & 0 & - \\
\hline $\begin{array}{l}\text { Joint drain } 32 \text { at Outlook Road } \\
\text { near Sunnyside, WA }\end{array}$ & 2.33 & 14.3 & 4.33 & 7 & 2 & - \\
\hline $\begin{array}{l}\text { Joint drain } 40.2 \text { near Tear Road } \\
\text { near Sunnyside, WA }\end{array}$ & 7.58 & 13.9 & 8.32 & 7 & 3 & - \\
\hline $\begin{array}{l}\text { Joint drain at Yakima Valley } \\
\text { Highway at Granger, WA }\end{array}$ & 3.49 & 10.9 & 5.46 & 7 & 3 & - \\
\hline $\begin{array}{l}\text { Joint drain from Rougk Lane near } \\
\text { Sunnyside, WA }\end{array}$ & 1.35 & 9.06 & 4.09 & 7 & 0 & - \\
\hline $\begin{array}{l}\text { Joint drain near S. 1st Street at } \\
\text { Sunnyside, WA }\end{array}$ & 1.97 & 7.78 & 4.74 & 7 & 0 & - \\
\hline $\begin{array}{l}\text { Roza Canal Wasteway Number } 3 \\
\text { near Sawyer, WA }\end{array}$ & $<0.040$ & 0.072 & $<0.040$ & 5 & 0 & - \\
\hline $\begin{array}{l}\text { Sulphur Creek Wasteway at } \\
\text { Sheller Road at Sunnyside WA }\end{array}$ & $<0.040$ & 0.242 & 0.075 & 5 & 0 & - \\
\hline $\begin{array}{l}\text { Sulphur Creek Wasteway near } \\
\text { Sunnyside, WA }\end{array}$ & 2.4 & 9.2 & 3.91 & 7 & 0 & - \\
\hline \multirow[t]{2}{*}{ Washout Drain at Sunnyside, WA } & 5.7 & 11 & 8.3 & 7 & 2 & - \\
\hline & & & Total & 164 & 21 & - \\
\hline
\end{tabular}




\section{Summary}

Nitrate contamination in drinking water of the lower Yakima River Basin Groundwater Management Area (GWMA) is a major concern for local, state and federal agencies. During April through December 2017, the U.S. Geological Survey (USGS) conducted a large sampling collection effort, consisting of the collection of samples (892) from 156 domestic drinking water wells, and 164 samples from 24 drains sites, to fill identified nitrate concentration data gaps and help establish a baseline condition of nitrate concentrations for future assessments within the GWMA.

Domestic well sample locations were selected based on owner permission, ability to collect a representative sample and spatial distribution across the study area. Drains sites were selected based on distribution across the GWMA, and ability to access the site and collect a representative sample.

Sampling collection, processing, and field analyses were in accordance with applicable USGS procedures and included thorough well purging, use of non-contaminating equipment, and quality-assurance sampling. Quality assurance samples made up 8 percent of all samples and included blanks and replicates. No changes were made to the dataset based on these quality-assurance data.

The mean nitrate concentration collected from domestic wells and drains was 6.1 milligrams per liter $(\mathrm{mg} / \mathrm{L})$ and $5.6 \mathrm{mg} / \mathrm{L}$, respectively. The $10 \mathrm{mg} / \mathrm{L}$ maximum contaminant level (MCL) was exceeded by 20 percent of samples from wells and 13 percent of samples from drains. At least one nitrate concentrations was detected above the (MCL) in 26 percent of wells and 33 percent of drains sampled nitrate was not detected $(<0.04 \mathrm{mg} / \mathrm{L})$ in 13 percent of all samples.

\section{References Cited}

Ely, D.M., Bachmann, M.P., and Vaccaro, J.J., 2011, Numerical simulation of groundwater flow for the Yakima River Basin aquifer system, Washington: U.S. Geological Survey Scientific Investigations Report 2011-5155, 90 p.

Jones, M.A., and Vaccaro, J.J., 2008, Extent and depth to top of basalt and interbed hydrogeologic units, Yakima River Basin aquifer system, Washington: U.S. Geological Survey Scientific Investigations Report 2008-5045, 22 p., 5 pls.

Jones, M.A., Vaccaro, J.J., and Watkins, A.M., 2006, Hydrogeologic framework of sedimentary deposits in six structural basins, Yakima River Basin, Washington: U.S. Geological Survey Scientific Investigations Report 20065116, 24 p., 7 pls.

Patton, C.J., and Kryskalla, J.R., 2011, Colorimetric determination of nitrate plus nitrite in water by enzymatic reduction, automated discrete analyzer methods: U.S. Geological Survey Techniques and Methods, book 5, chap. B8, 34 p.

U.S. Environmental Protection Agency, 2012, Relation between nitrate in water wells and potential sources in the lower Yakima Valley, Washington: U.S. Environmental Protection Agency, EPA-910-R-12-003.

U.S. Geological Survey, variously dated, National field manual for the collection of water-quality data: U.S. Geological Survey Techniques of Water-Resources Investigations, book 9, chaps. A1-A9, accessed March 15, 2016 at http://pubs. water.usgs.gov/twri9A.

Washington State Department of Ecology, 2010, Lower Yakima Valley groundwater quality preliminary assessment and recommendations document: Washington State Department of Ecology Publication No. 10-10-009. 
Publishing support provided by the U.S. Geological Survey

Science Publishing Network, Tacoma Publishing Service Center

For more information concerning the research in this report, contact the Director, Washington Water Science Center

U.S. Geological Survey

934 Broadway, Suite 300

Tacoma, Washington 98402

https://wa.water.usgs.gov 
